\title{
Dust Distribution in Gas Disks. A Model for the Ring Around HR 4796A
}

\author{
H.Hubertus Klahr \\ and \\ D.N.C. Lin \\ UCO/Lick Observatory, University of California, Santa Cruz, CA 95064
}

\begin{abstract}
There have been several model analyses of the near and mid IR flux from the circumstellar ring around HR4796A. In one set of models, the 10 and $18 \mu \mathrm{m}$ IR flux has been attributed to the reprocessing of stellar radiation by $\mu m$-size particles. Since these particles are being blown away, on a dynamical time-scale, by the radiation pressure of HR4796A, they must be continually replenished by the collisional fragments of larger particles. If the ring appearance persisted for the life span $\left(8 \times 10^{6} \mathrm{yr}\right)$ of HR4796A, a parent-particle reservoir with a total mass $>5 M_{\oplus}$ would be needed. In order to avoid being conspicuous at longer wavelengths, most of the mass must be contained in parent particles larger than $20-40 \mathrm{~cm}$. In other models, it has been suggested that the IR flux from the rings is emitted by sufficiently large particles that survive the radiative blow out by their host star. In a gas free ring, $10-20 \mu m$-size particles would survive radiative blow out and a total of $10^{-3} M_{\oplus}$ would be adequate to account for the observed IR flux. But, in the vicinity of a young star, the possibility that the dust ring is embedded within a residual protostellar gas disk cannot be ruled out. In a gas-rich environment, larger sizes $(>100 \mu \mathrm{m})$ are needed for the particles to survive the radiative blow out. The total dust mass required to account for the IR flux is $<10^{-2} M_{\oplus}$. The combined influence of gas and stellar radiation may also account for the observed sharp inner boundary and rapidly fading outer boundary of the ring. The pressure gradient induced by a small (10\%) amplitude variation in the surface density distribution of a low-mass gaseous disk would be sufficient to modify the rotation speed of the gas. The resulting hydrodynamic drag on modest-size $(>100 \mu \mathrm{m})$ particles would be adequate to compensate for the turbulent stirring, radiative drag and radiation pressure such that they remain gravitationally bound to the system. The required surface density variation of the gas may be induced by 1) the perturbation of a low-mass planet or the binary companion HR 4796B, 2) the photo evaporation of the disk, or 3) from the variations in the viscous angular momentum transport and mass diffusion rate in the disk. We show that the structure of the dust ring is preserved during and after the gas is being depleted such that similar rings may be common among early type stars.
\end{abstract}


Subject headings: circumstellar matter, planetary systems, stars

\section{Introduction}

The observational discoveries (Smith \& Terrile 1984) of $\beta$ Pic type dusty rings provide tantalizing hints of residual planetesimal disks similar to the Kuiper belt may exist around other stars (Artymowicz 1997). Perhaps the most intriguing dust rings are those around HR 4796A (Schneider et al. 1999) and HD 141569 (Weinberger et al. 1999). The sharp surface density variations near the ring boundaries of HR 4796A are remarkably similar to that found around Saturn's F ring (Cuzzi et al. 1984) and Uranus' $\epsilon$ ring (Elliot \& Nicholson 1984). Under the action of viscous stress, these differentially rotating disks have a general tendency to diffuse in the radial direction (Lynden-Bell \& Pringle 1974). The expansion of planetary rings are prevented by the tidal torques of nearby shepherding satellites (Goldreich \& Tremaine 1979, 1982). The structural resemblance between circumstellar and planetary rings naturally leads to the conjecture that the former, too, may be perturbed by nearby hypothetical planets (Jura et al. 1995). The reported azimuthal brightness asymmetry (Telesco et al. 2000) provides a supporting evidence for the scenario that the ring may be gravitationally perturbed by a planetary or stellar companion (Wyatt et al. 2000).

Another motivation for invoking the embedded planet scenario is the perseverance of the ring itself. The NICMOS image shows that the surface brightness has a maximum at 70 AU with a sharp decline at the smaller radii and an inner cutoff near 50 AU. Outside this region, the surface brightness decreases more gradually with radius to less than half of the peak brightness at 90AU (Schneider et al. 1999). The observed ratio of flux from the ring to that from the star increases with wavelength at 1.1 and $2.6 \mu \mathrm{m}$, indicating the mean particle size is larger than these wavelength. But, using the flux ratio at 10 and $18 \mu \mathrm{m}$ and assuming a Mie emissivity, Telesco et al. (2000) inferred a "characteristic" diameter for the dust particles to $\sim 2-3 \mu \mathrm{m}$ which is in contrast to the lower particle-size limit of $10 \mu \mathrm{m}$ inferred from the $60 \mu \mathrm{m}$ radiation by Jura (et al. 1993). The observed spectral energy distribution (SED) with wavelength $\lambda=10-10^{3} \mu m$ (Koerner et al. 1998) has been modeled. Using single-size particles $(>100 \mu m)$, Augereau et al. (1998) were able to fit the observed SED between $10-10^{2} \mu \mathrm{m}$ but not the resolved images of the ring. Both the SED and the resolved images were better modeled with reprocessing due to particles with a power-law collisional particle-size distribution (Heller 1970, Mathis et al. 1977) with adopted minimum $\left(a_{\min }\right)$ and maximum $\left(a_{\max }\right)$ sizes of $\sim 10 \mu \mathrm{m}$ and a few meters respectively. In these models, since the models are applied to SED in the wavelength range of $10-10^{3} \mu \mathrm{m}$, the actual values chosen for $a_{\min }$ and $a_{\max }$ do not significantly affect the actual match.

With the stellar luminosity $L_{\star}=35 L_{\odot}$, small particles, if they exist, would be blown away by the radiation pressure on the time-scale of a few hundred years (Backman \& Paresce 1993). In the models adopted by Augereau et al. (1998), all particles with sizes larger than $a_{\text {min }}$ would survive the radiative blow-out effect. But for the models proposed by Telesco et al. (2000), the 
"characteristic-size" particles would not be able to remain. In a companion paper, Wyatt et al. (2000) suggested that these small particles are probably the collisional fragment of a population of larger particles. In this replenishment scenario, it is still essential for the parent particles to have an adequate reservoir of total mass to replenish the $\mu m$-size particles for HR 4796A's life span $\tau_{\star}=8 \times 10^{6}$ yr (Stauffer et al. 1995, Jayawardhana et al. 1998). In order to be consistent with the observed SED and ring structure, most of these parent particles must also be located within $70 \pm 20 \mathrm{AU}$ and have a surface density comparable to or less than that inferred by Augereau et al. (1998). One or more nearby planets may provide both the supply and confinement mechanism for the parent particles.

An important issue to be resolved is the required mass for such perturbers. In HR 4796A, such confining planets must either form or migrate to the vicinity of the rings (which is well beyond the orbit of Neptune) within $\tau_{\star}$. In conventional theories, the first stage of planetary formation is the emergence of planetesimals and solid cores (Safronov 1969; Wetherill 1980). Only after these cores have acquired a few Earth-masses, dynamical accretion of gas becomes possible (Pollack et al. 1996). But, beyond the orbit of Neptune, the formation of a few Earth masses core requires at least several $10^{7} \mathrm{yr}\left(>\tau_{\star}\right)$ unless the mass of solid material is much larger than that inferred from the minimum mass solar nebula model (Lissauer 1993). Assuming an initial surface density 10-20 times that of the minimum mass solar nebula (which corresponds to 150-300 $M_{\oplus}$ within the annulus between $\sim 58-82 \mathrm{AU}$ ), Kenyon et al. (1999) found it possible to form planetesimals larger than $10^{3} \mathrm{~km}$ within $10^{7} \mathrm{yr}$. They further suggested that gravitational perturbation by these large objects may excite large velocity dispersion which may lead disruptive collisions. However, if this assumed surface density of the dust particles is augmented for the solar composition, the inferred mass of the gaseous ring $\left(\sim 0.05-0.1 M_{\odot}\right.$ within $\left.70 \pm 12 \mathrm{AU}\right)$ would make it gravitationally unstable. Cameron (1978) and Boss (1998) have suggested that gravitational instability in the disk may indeed lead to the formation of giant planets directly from the disk gas. But, some numerical simulations show that the growth of non axisymmetric perturbations excited by gravitational instability are more likely to induce efficient transfer of angular momentum in the disk rather than fragmentation (Nelson et al. 1998, Burkert \& Bodenheimer 1996). The initial surface density required for this conjecture is also large compared with the mass inferred for the outer regions of typical protostellar disks (Beckwith \& Sargent 1993).

In this paper, we examine the persistence of the dust ring structure around HR 4796A. Since the host star is only $8 \times 10^{6} \mathrm{yrs}$ old, we hypothesize the existence of some residual gas analogous to those found around several young stellar objects with similar ages (Beckwith et al. 1990, Beckwith \& Sargent 1993, Zuckerman et al. 1995, Hartmann et al. 1998). An upper limit 1-7 $M_{\oplus}$ of gas has been inferred from the mm continuum radiation by Greaves et al. (1999). (A larger gas mass may exist if a substantial amount of heavy element is depleted from the gas phase and is stored in particles larger than a few mm.) We explore the interaction between the dust, radiation, and residual gas in the disk. In $\S 2$, we briefly recapitulate the physical interaction between particles, stellar radiation, and residual gas. We show that the hydrodynamic drag tends to promote the 
effect of radiation pressure and increase the critical particle size for radiative blow out. In $\S 3$, we provide some constraints on the size and total mass of particles in the ring around HR 4796A. We show that variations in the surface distribution of the gas provide a favorable condition for modest (more than $100 \mu \mathrm{m}$ ) size particles to congregate into a dust ring. This ring structure is preserved during and after the depletion of the disk gas. We suggest that these particles provide a supply of the dominant contributors to the IR emission of the dust ring. Finally in $\S 4$, we discuss the implications of these results on planet formation and the ubiquity of similar rings around other early type stars.

\section{The Dynamics of Residual Dust Particles}

In this section, we examine the interaction between stellar radiation, residual disk gas, and dust particles of various sizes.

\subsection{The gaseous disk}

For illustrative purpose, we consider a simple model in which a disk of dust particles is embedded in a residual gaseous disk. The only free parameters in this model are those which describe the gas surface density $\Sigma(R)$ as a function of the disk radius $R$. All other gas properties are deduced in a self-consistent way.

The qualitative results presented here depend on the gross global gas distribution in the disk and are insensitive to the detailed expression of $\Sigma(R)$. In principle, the $\Sigma$ distribution is determined by the efficiency of angular momentum transfer (e.g. via viscosity from turbulence) in the disk and the tidal perturbation by planetary or stellar companions. The former mechanism generally leads $\Sigma$ to attain a power law function of $R$ in most regions of the disk (Lin \& Bodenheimer 1981; Ruden \& Lin 1986). The latter process leads to a localized $\Sigma$ variation such as gaps (Lin \& Papaloizou 1979; 1993) and confined rings (Goldreich \& Tremaine 1978). Although low-mass companions may not be able to induce the formation of a gap, they can nevertheless introduce variations in the surface density of the disk. Self-gravitational effects will not affect the disk structure as the surface density in our models is always orders of magnitude smaller than any critical value (see Toomre 1964). For computational purpose (e.g. to produce our plots), we adopt the following generic prescription in which the global variation of $\Sigma$ is non monotonic:

$$
\Sigma=\Sigma_{0} \times\left(\begin{array}{clc}
d\left(\frac{R}{R_{0}}\right)^{-2.5} & \text { if }: & R<R_{1} \\
e^{-\frac{\left(R-R_{0}\right)^{2}}{\delta R_{0}^{2}}} & \text { if }: & R_{1} \leq R \leq R_{0} \\
\left(\frac{R}{R_{0}}\right)^{-2.5} & \text { if }: & R>R_{0}
\end{array}\right)
$$

where $d$ is a model parameter which determines the amplitude of a local maximum. The magnitude 
of $\Sigma$ at some fiducial radial location $R_{0}$ is set to be $\Sigma_{0}$. For $R<R_{1}$ and $R>R_{0}, \Sigma$ decreases monotonically with $R$ following a power law (with an index -2.5 ) which is somewhat steeper than that for the minimum mass solar nebula model (Hayashi et al. 1985). But in the outer region of the disk, $\Sigma$ is likely to decline more rapidly with $R$ as inferred from observation (Mundy et al. 1996, Hartmann et al. 1998). Since our results are insensitive to the detailed nature of the global radial dependence of $\Sigma$, we choose such a power law so that the disk mass remains finite at large $R$.

The inner and outer regions of the disk are connected through a transition zone at $R_{1} \leq R \leq R_{0}$. In this zone the surface density has the shape of a Gaussian with a width of $\delta R_{0}$. The value of $R_{1}$ is set to satisfy $\left(R_{1}-R_{0}\right)=\delta R_{0}\left(2.5 \ln \left(R_{1} / R_{0}\right)-\ln d\right)^{1 / 2}$ such that $\Sigma$ is continuous. In all our models, we choose $d<1$ which is reasonable because the continuum flux in the mid-IR wavelength range is observed to be more intense than that in the near-IR range in several protostellar disks (Hillenbrand et al. 1998). One possible interpretation is that these disks are more depleted in the inner region or better preserved in the outer region as in the context of circumbinary disks (Jensen \& Mathieu 1997). On the theoretical side, such a $\Sigma$ variation is expected if a) the viscosity decreases rapidly with radius (Ruden \& Pollack 1991), b) the inner region is depleted by either a stellar or a disk wind (Shu et al. 1994, Konigl \& Ruden 1993), c) HR4796A is a A0V star and a strong source of UV radiation which could lead to photo evaporation in some regions of the disk (Shu et al. 1993) and quench gas infall along the rotation axis and onto the inner regions of the disk (Yorke et al. 1995), d) the disk is depleted by the formation of one or more planets which are expected to first emerge in the inner regions of the disk (Jura et al. 1993), or e) the structure of disk around HR4796A may be tidally perturbed by the stellar companion HR4796B (Augereau et al. 1998), especially if the binary orbit is eccentric (Korycansky \& Papaloizou 1995). The difference between the inner and outer regions of the disk can be minimized by setting $d$ close to unity. Finally, the $\Sigma$ variation in the transition region $R_{1} \leq R \leq R_{0}$ can also represent a tidal perturbation of a nearby embedded planetary companion.

The value of $\Sigma_{0}$ is set such that the mass $M_{g}=2 \pi \int_{R_{0}-\delta R_{0}}^{R_{0}+\delta R_{0}} R \Sigma(R) d R$ contained within a ring centered on $R_{0}$ with a half width $\delta R_{0}$ is to be specified as a model parameter. This mass is to be spread over a ring with a half width $\delta R_{0}=10-20 \mathrm{AU}$ centered on a radial location $R_{0}=70 \mathrm{AU}$. In our models $M_{g}$ is 1-100 Earth masses $\left(M_{\oplus}\right)$. The corresponding $\Sigma$ is orders of magnitude smaller than that for the minimum mass solar nebula model at a similar distance from the central star (Hayashi et al. 1985).

The outer regions of the disk are optically thin such that every dust particle in the disk receives direct irradiation from the central object. The particles are heated to the local black body temperature (small grains may be hotter because they cannot radiate efficiently, Chiang \& Goldreich 1997). The luminosity and mass of HR 4796A are $L_{\star}=35 L_{\odot}$ and $M_{\star}=2.5 M_{\odot}$ respectively (Jura et al. 1993). The temperature of the dust grains can be given by:

$$
T_{d}=\left(\frac{L_{\star}}{16 \pi a R^{2}}\right)^{1 / 4}
$$


where $a$ is the radiation density constant. This upper limit estimation holds for particles larger than the typical wave length of the radiation and assuming an albedo of zero. A more careful treatment of the optical properties of the particles is not necessary in the context of the effects studied in this paper.

If gas molecules collide frequently with the dust particles, they can also attain a similar temperature, $T_{h i g h}=T_{d}$. In the low gas density limit, however, thermal conduction between the dust and the gas is less efficient than the gas radiative processes. As a consequence of this thermal imbalance, the gas temperature may decrease to a minimum value $\left(T_{\text {low }}=10 \mathrm{~K}\right)$ which is comparable to that of the radiation background field (Preibisch, Sonnhalter and Yorke 1995; Yorke \& Lin in preparation). In general, $T_{\text {low }}<T_{\text {gas }}<T_{\text {high }}$ and the associated sound speed $c_{s}=\sqrt{T_{\text {gas }} * R_{\text {gas }} / \mu}$, where $R_{\text {gas }}$ is the gas constant and $\mu$ the mean molecular weight (i.e. $\mu=2.353$ for an $\mathrm{He}-\mathrm{H}_{2}$ mixture). The disk thickness is determined by its pressure scale height $H_{p}=c_{s} / \Omega$ where $\Omega \equiv\left(G M_{*} / R^{3}\right)^{1 / 2}$ is the Keplerian frequency. At $R=70$ AU, $0.03<H_{p} / R<0.1$ for the low and high temperature limits. For the parameters we have adopted, the disk gas is likely to be in the low temperature and small thickness range. The local gas density in the midplane of the disk can be estimated as $\rho=\Sigma / 2 H_{p}$ and the pressure $p$ becomes $p=c_{s}^{2} \rho$.

The azimuthal orbital frequency of the gas $(\omega)$ is given by the condition for hydrostatic equilibrium between the gravitational potential of the central object and the sum of centrifugal acceleration and the radial pressure gradient

$$
\frac{G M_{\star}}{R^{2}}=\omega^{2} R+\frac{1}{\rho} \nabla p
$$

which leads, in first order approximation, to a local deviation $d V$ from the Keplerian profile of (see Whipple 1972)

$$
d V \equiv(\omega-\Omega) R=V_{\phi}-\Omega R=\frac{1}{2 \Omega \rho} \nabla p \simeq \frac{c_{s}^{2}}{2 \Omega R} \frac{\partial \ln \rho}{\partial \ln R}
$$

where $V_{\phi}=\omega R$ is the azimuthal speed. In $\S 2.3$, we consider the case that this angular frequency may be modified by the particles' drag when they become the dominant constituent of the disk.

Results under specific assumptions for the free parameters of this model will be discussed in the $\S 3$.

\subsection{Radial drift of dust grains}

The particles are drifting radially under two independent physical effects: 1) hydrodynamic drag by the gas and 2) the radiation pressure of photons emitted by the central object. 


\subsubsection{Departure from Keplerian flow}

While the pressure gradient induces the gas to attain a finite $d V$, the particles' motion is determined by the gravity of the central star, the hydrodynamic drag by the disk gas, and the drag and pressure by the stellar radiation. Radial inward and outward drift of the particles can be generated by the non-Keplerian motion of the gas. Small particles are well coupled to the gas, thus they also move on non-Keplerian orbits provided their Stokes number $S t \equiv \tau_{f} \Omega<<1.0$ (Weidenschilling 1977). The friction time for spherical particles in the Epstein regime (e.g. Klahr \& Henning 1997) is given by

$$
\tau_{f}=\frac{a_{d} \rho_{d}}{c_{s} \rho}=\frac{3 m_{d}}{4 A_{d} c_{s} \rho}
$$

where $a_{d}$ is the particles radius, $m_{d}$ its mass, $A_{d}=\pi a_{d}^{2}$ its cross section, $\rho_{d}$ its density, $c_{s}$ the thermal velocity of the gas and $\rho$ the local gas density. In the case of transonic velocities (which is

irrelevant in our model) $c_{s}$ has to be replaced by $\sqrt{c_{s}^{2}+d v^{2}}$ with the relative velocity $(d v)$ between the particle and the gas (H. Yorke 1999, private communication). The critical particle size which demarcates the small and large particle size range then becomes

$$
a_{0}=\Sigma / 2 \rho_{d}
$$

which is a few $\mathrm{cm}$ for a minimum mass solar nebula model at $70 \mathrm{AU}$ and smaller for a depleted gaseous disk. In our standard models (see Sect. 3.5.1 and Sect. 3.5.2 ), $a_{0}=0.06 \mathrm{~cm}$ at $R_{0}$.

In the solar system today, the absence of gas makes $S t>>1$ for all particles. Modest-size particles spiral inwards due to the Poynting Robertson effect. But, spherical particles (with $\rho_{d}=2.5 \mathrm{gm} \mathrm{cm}^{-3}$ ) with size

$$
a_{d}<a_{1} \equiv \frac{3}{16 \pi} \frac{L_{\star}}{G M_{\star} c \rho_{d}} \sim 3.2 \mu m
$$

would be blown out by the radiation pressure on hyperbolic orbits as "beta meteorites" (see Grün et al. 1985) on a time-scale comparable to the local dynamical time-scale $\tau_{d} \sim \Omega^{-1} \sim 10^{2}$ yr (see below). For our considerations we neglect particles smaller than the typical wavelength of the radiation (No Mie scattering).

Larger particles would remain bound to the host star. They can even remain on a circular orbit with a sub Keplerian azimuthal velocity

$$
v_{\phi}^{*}=\sqrt{\frac{G M_{\star}-\frac{L_{\star} A_{d}}{4 \pi m_{d} c}}{R}},
$$

where $v_{\phi}^{*}$ denotes the particles theoretical velocity in the absence of gas, which is an important quantity (see below). While the particles are orbiting around the central star they also experience a radiative head wind due to the aberration of its photons. In gas-free environments, such as our own solar system, this Poynting-Robertson radiative drag effect induces the azimuthal velocity $\left(v_{\phi}\right)$ of small particles to change at a rate

$$
\dot{v}_{\phi}=-\frac{L_{\star}}{4 \pi R^{2}} \frac{A_{d}}{m_{d} c^{2}} v_{\phi} .
$$


This process leads an inward drift for all remaining particles with $a_{d}>a_{1}$.

In a gaseous environment, the combined influence of hydrodynamic and radiative drag induces the particles' azimuthal velocity to change at a rate

$$
\dot{v}_{\phi}=-\frac{\left(v_{\phi}-V_{\phi}\right)}{\tau_{f}}-\frac{L_{\star}}{4 \pi R^{2}} \frac{A_{d}}{m_{d} c^{2}} v_{\phi} .
$$

If $\tau_{f}$ is small in comparison to the relevant evolutionary time-scales (see Fig. 11 and Fig. 3), an equilibrium would be rapidly established with $\dot{v}_{\phi}=0$ so that

$$
v_{\phi}=\frac{V_{\phi}}{1+\tau_{f}\left(\frac{L_{\star}}{4 \pi R^{2}} \frac{A_{d}}{m_{d} c^{2}}\right)} .
$$

Substituting $\tau_{f}$, we find

$$
v_{\phi}=\frac{V_{\phi}}{1+\left(\frac{L_{\star}}{4 \pi R^{2}} \frac{3}{4 c^{2} c_{s} \rho}\right)}
$$

which is independent of particle sizes (as long as $S t<<1$ and the particles larger than the wavelength of the radiation)!

Around young stellar objects where the particles are embedded in a gaseous circumstellar disk environment, the term $d v \equiv v_{\phi}-V_{\phi}$ (see Fig. 1) is typically less than of $1 \mathrm{~mm} / \mathrm{s}$ at $70 \mathrm{AU}$ while $d V \sim 10^{3} \mathrm{~cm} / \mathrm{s}$. Note that the Poynting Robertson effect is insignificant until $\Sigma$ is depleted below $3 L_{\star} / 8 \pi R^{2} c^{2} \Omega$ which is not only more than four orders of magnitude below that for our standard model and that inferred from $\mathrm{mm}$ continuum radiation for typical protostellar disks but also smaller than that inferred for the CO gas around some young stellar objects (Zuckerman et al. 1995). (The actual gas content may be even larger if a significant amount of $\mathrm{CO}$ gas is depleted due to grain condensation.) Thus, in our estimate of the particles' motion, we only consider contributions from $d V$ and neglect the Poynting Robertson effect.

\subsubsection{Radial drift}

In addition to the radiative drag in the azimuthal direction, the stellar photons also exert on the particles a radiative pressure in the radial direction. In general, the radial drift $v_{r}$ of small particles (with $S t<1$ ) results from the non-Keplerian motion and the radial radiation pressure can be obtained from the radial component of the equation of motion such that

$$
\frac{\partial v_{r}}{\partial t}=-\frac{v_{r}}{\tau_{f}}+\frac{v_{\phi}^{2}}{R}-\frac{G M_{\star}}{R^{2}}+\frac{L_{\star}}{4 \pi R^{2}} \frac{A_{d}}{m_{d} c} .
$$

In order to simplify work we replace $\Omega$ by $\Omega^{*}\left(\equiv v_{\phi}^{*} / R\right)$ which is the orbital frequency with respect to the radial radiation pressure (see Eq. 8 ). Note that $\Omega^{*}<\Omega$ in general. Thus it follows

$$
\frac{\partial v_{r}}{\partial t}=-\frac{v_{r}}{\tau_{f}}+\frac{\left(\Omega^{*} R+d V^{*}\right)^{2}}{R}-\Omega^{* 2} R .
$$


Here $d V^{*} \equiv v_{\phi}-\Omega^{*} R$ denotes the deviation of the gas from the modified Keplerian orbit. Since the radius dependence of gravity and radiation pressure is identical, the outward radiation pressure effectively reduces the gravitational attraction on the particles imposed by the host star everywhere (Gustafson 1994).

The "characteristic size" of particles inferred by Telesco et al. (2000) is smaller than $a_{1}$ such that they are expected to be blown away by the radiation pressure of HR4796A. In order to remove this dilemma, Wyatt et al. (1999) suggested that although these small particles are continually being blown out, they are also being replenished by collisional fragmentation of larger particles which are not significantly affected by the radiation pressure. In a gas free environment, the parent body size must be larger than $a_{1}$.

But, around many young stellar objects, small particles are embedded in a residual gaseous background. The gas generally does not directly experience the radiation pressure because its molecular opacity is too small. Thus, the gas azimuthal speed $\left(V_{\phi}\right)$ is independent of $L_{\star}$ and it is given by eq. (3). The discussions in $\S 2.2 .1$ indicate that particles with $S t<1$ (or equivalently with $a_{d}<a_{0}$ ) have $v_{\phi} \sim V_{\phi}$ (see eq. 12). As the hydrodynamic drag forces these small particles to nearly corotate with the gas, they attain a finite $v_{r}$ as a consequence of being out of hydrostatic equilibrium. When a steady state is established,

$$
v_{r}=\tau_{f} 2 \Omega^{*} d V^{*} \text { for } S t<<1,
$$

in the limit that the quadratic term $d v^{2}$ is small in comparison to $\Omega^{*} R d v^{*}$ (cf Weidenschilling 1977; He used another definition of $d V$ !). The sign of $d V^{*}$ can now be negative or positive, depending on whether the particle is less or even more sub-Keplerian than the gas. Relatively large particles follow in a similar fashion,

$$
v_{r}=2 d V^{*} \quad \text { for } \quad S t=1
$$

and

$$
v_{r}=\frac{2 d V^{*}}{\Omega^{*} \tau_{f}} \quad \text { for } \quad S t>>1
$$

Note that the effect of radiation pressure decreases with the particle size. From this equation one can deduce that in regions where $d V>0$ (as a result of a negative gas pressure gradient), all the particles with would migrate outwards since their azimuthal speed is always slightly hyper-Keplerian, just like the gas. But in the more common situation where $d V \leq 0$ (which corresponds to a negative gas pressure gradient as expected in unperturbed regions of protostellar disks), hydrodynamic drag is more important than radiative drag for all particles with

$$
a_{d}>a_{2} \equiv-\frac{3 L_{\star}}{32 \pi R^{2} c \Omega \rho_{d} d V}
$$

so that they undergo orbital decay. In contrast, particles with $a_{d}<a_{2}$ are blown away by the stellar radiation pressure (see Fig. 目 and Fig. 3). This minimal size for the given parameters of HR $4796 \mathrm{~A}$ is $a_{2} \sim 100-200 \mu \mathrm{m}$ for almost all disk radii $R$. Only at those location where variations in 
$\Sigma$ lead to a reduction in $d V, a_{2}>500 \mu \mathrm{m}$. In the location where $d V$ vanishes or attains a negative value, $a_{2}$ becomes infinite and particles of all sizes (including planets) are prevented from inward radial drift.

We note that the magnitude of $a_{2}$ is larger than $a_{1}$ in eq(7) by a factor $\sim\left(R^{2} / H_{p}^{2}\right)(d \ln \rho / d \ln R)^{-1} \sim 10-100$ because the small particles are forced to corotate with the gas such that a centrifugal balance is always maintained. Thus, if they are embedded in a residual gaseous disk, the "characteristic-size" ( $\mu m$-size) particles in HR 4796A inferred by Telesco et al. (2000) must be supplied by parent bodies which are at least two order of magnitude larger than them.

In a gaseous disk environment, all particles with $a_{d}$ smaller than both $a_{0}$ and $a_{2}$ are blown away by the radiation pressure on the dynamical time-scale $\tau_{d}$. In the limit that $a_{0}>a_{2}$, particles with sizes $a_{0}>a_{d}>a_{2}$ can achieve both positive and negative radial drift velocities depending on the local gas pressure gradient. In $\S 3$, we present numerical results to show that these changes in the direction of particles' radial drift lead to the particles' accumulation into rings. Note that the value of $a_{2}$ does not directly depend on $\Sigma$. As the disk gas is depleted, neither $d V$ nor $a_{2}$ are strongly affected. But, $a_{0}$ is linearly proportional to $\Sigma$. When $a_{0}$ decreases below $a_{d}$ of some particles, they decouple from the gas and their azimuthal velocity becomes closer to the local modified Keplerian value $v_{\phi}^{*}$ (see eq. 8). Since their $a_{d}$ is larger than $a_{2}$ initially and $a_{2}$ decreases, the radiation pressure and the Poynting Robertson effect have a weaker effect than the hydrodynamic drag throughout the evolution such that their radial drift velocity is described by eq. 17. Eventually, the depletion of the residual gas increases $\tau_{f}$ and reduces $v_{r}$.

\subsection{Constrains on the disk profile}

We already noted that the trapping mechanism for particles is located at places, where the disk becomes Keplerian, as the radial pressure gradient vanishes, i.e.

$$
\nabla p=\nabla\left(c_{s}^{2} \rho\right)=\nabla\left(c_{s} \Sigma \Omega\right)=0 .
$$

Since $\Omega \sim R^{-3 / 2}$, the general criterion for the ring formation is

$$
c_{s} \Sigma \sim R^{3 / 2}
$$

This criterion can now be used to determine the local shape of the Surface density profile. We consider three distinct cases: 1$)$ an isothermal $\left.\operatorname{disk}\left(T=T_{\text {low }}\right), 2\right)$ an optically thin disk in radiation equilibrium $\left(T=T_{h i g h}\right)$, and 3$)$ an optically thick viscous disk.

In the isothermal case $c_{s}$ is constant such that the trapping mechanism requires $\Sigma$ to increase locally as $R^{3 / 2}$ regardless its magnitude and radial extent. In the standard optical thin case,

(perhaps most relevant to the case of HR4796A), $c_{s} \sim R^{-\frac{1}{4}}$ as $T \sim R^{-\frac{1}{2}}$. The necessary power-law $R$-dependence in $\Sigma$ is only slightly steeper than in the previous case: i.e. $\Sigma \sim R^{\frac{7}{4}}$. 
In an optically thick disk which is heated by the local viscous dissipation, the disk's mid plane temperature $T_{c}=T_{e}\left(3 \tau_{r} / 8\right)^{1 / 4}$ where the vertical optical thickness $\tau_{r} \approx \kappa \Sigma / 2$ and the Rosseland mean opacity $\kappa=\kappa_{o} T_{c}^{2}$ (Lin \& Papaloizou 1985). If the disk is approximately in a steady state, its effective temperature $T_{e} \propto R^{-\frac{3}{4}}$ (e.g. Lynden-Bell \& Pringle 1974, Bell et al. 1997) such that $T_{c} \propto \kappa_{o}^{1 / 2} T_{e}^{2} \Sigma^{1 / 2} \propto R^{-3 / 2} \Sigma^{1 / 2}$. Particles are trapped in regions where $\Sigma \propto \kappa_{o}^{-1 / 5} R^{9 / 5}$ which is similar to the isothermal and the optically thin cases.

The dominant contributors to the disk opacity are grains. Across some critical locations (dust destruction zone) in the disk where the grains sublimate, the opacity increases mainly through changes in the scaling constant $\kappa_{o}$ (Ruden \& Lin 1986). Particles may be trapped in regions where $\kappa_{o} \propto R^{9}$. A full 2-D detailed model is needed to address the issue whether such a transition may indeed be a viable effect to prevent dust in form of meter sized solid bodies from drifting into the sun and help collect bodies at certain transition radii from the sun to form the terrestrial planets. For the present context, the ring around HR $4796 \mathrm{~A}$ is well outside the ice and silicate destruction zones.

\subsection{Particle-dominant flow}

In the above discussion, we neglect the feedback effect of hydrodynamic drag on the gas flow. In principle, the gas flow may be perturbed by the motion of the particles when the particles dominate in mass surface density $\left(\Sigma_{d}\right)$ over the surrounding gas (Nakagawa 1986). In the Kuiper Belt today, no sign of residual gas has been detected. Gas may be preferentially depleted prior to the depletion of the dust particles. When the surface density of the gas $\Sigma$ decreases below that of the dust $\Sigma_{d}$, the hydrodynamic drag between them may induce the gas to comove with the particles rather than forcing the particles to corotate with the gas. In reality, however, the only particles which can provide an efficient momentum transfer with the gas are those with sizes $a_{d}<<a_{0}$ (Nakagawa 1986). During the depletion of gas, $a_{0}$ decreases with $\Sigma$, leading to a reduction in the total fraction of particles which are well coupled with the gas. Thus, the gas speed is unlikely to be significantly affected by the particles' drag effect.

For those regions of the disk with $\Sigma>\Sigma_{d}$, gas flow would be affected by the hydrodynamic drag of the particles near the midplane of the disk if the total mass density of the particle $n_{d} m_{d}$ exceeds the gas density $\rho \sim \Sigma / H_{p}$ (Weidenschilling \& Cuzzi 1993). (Similarly, particle accumulation due to variations in the radial drift speed may also lead to local enhancement of particle density.) The average mass density of the particles $\rho_{\text {dust }}$ is determined by their scale height $H_{d}=\sigma_{d} / \Omega$ where $\sigma_{d}$ is the velocity dispersion of the particles. In a turbulent medium, the particles are being stirred by the dispersive motion of the gas. Since the turbulent speed of the gas

is unlikely to exceed its sound speed, particles with $a_{d}>a_{0}$ would sediment toward the mid plane and attain a scale height $H_{d}<H$. With a substantially sub sonic turbulent speed, sedimentation is also possible for sufficiently small $\left(a_{d}<a_{0}\right)$ particles which couple well with the gas. Only after $\rho_{\text {dust }}$ of the small $\left(a_{d}<a_{0}\right)$ particles has exceeded the gas density $\sim \Sigma / 2 H$, would the gas motion 
be forced to corotate with the small particles' $\Omega^{*}$ of the rather than $\omega$. Without a significant differential motion between them and the gas, particles with $a_{1}<a_{d}<a_{0}$ would not undergo any further orbital migration. But larger $\left(a_{d}>a_{0}\right)$ particles are less affected by the radiation pressure and have a larger $v_{\phi}^{*}$. Gas drag induced by the residual headwind would continue to drive the larger particles inward though at a much reduced pace.

But, the optical depth of HR4796 A is well below unity and

$$
\rho_{\text {dust }}=\frac{2 L_{\text {disk }}}{3 \pi L_{*}} \frac{\rho_{d} a_{d}}{H_{d}}
$$

(see eq. 26 below). For particles of $a_{d}=200 \mu \mathrm{m}$ the density is $\rho_{\text {dust }}<<6.3 \times 10^{-17} \mathrm{~g} \mathrm{~cm}^{-3}$, which is well below the gas density inferred from the minimum mass solar nebula model. For those models we have adopted in this paper, particles have negligible drag effect on the motion of the gas.

\section{Models for HR 4796A}

In this section, we present a scenario for the ring structure around HR 4796A. First, we revisit the two existing models for particle size distribution by analyzing the preferred size of particles for retention. We describe a simple quasi static model in which we assume that HR 4796A had initially a circumstellar gas and dust disk around it. We then show that a dust disk will evolve into a ring structure with sharp edges. We argue such a structure would be preserved after the gas is depleted.

\subsection{Replenishment of a population of $\mu m$-size particles}

First, we examine the implication of the scenario proposed by Telesco et al. (2000) in which the dominant contributors to the 10 and $18 \mu \mathrm{m}$ radiation from the ring around HR 4796A are $\mu m$-size particles. Since the "characteristic" size of the particle is comparable to the wavelength of the photon, the reprocessed radiation by the disk particles

$$
L_{d i s k}=Q L_{\star} \frac{\sigma_{d}}{2 R d R} \frac{M_{\text {char }}}{m_{d}}
$$

where $Q$ is the albedo and assumed to be one for large particles and $d R$ the radial width of the dust ring. Assuming $\mathrm{SiO}$-dust particles which have spherical shape and a density $\rho_{d}=2.5 \mathrm{gm}$ $\mathrm{cm}^{-3}$, the total mass of those particles with "characteristic" sizes is

$$
M_{\text {char }}=\frac{8}{3} \frac{L_{\text {disk }}}{L_{\star}} R d R \rho_{d} \times a_{d} .
$$

The total IR luminosity of the ring is estimated to be $L_{d i s k} / L_{\star}=5 \times 10^{-3}$ (Jura et al. 1993) so that

$$
M_{\text {char }}=\left(a_{d} / 1 \mathrm{~cm}\right) \times 1.83 \times 10^{29} \mathrm{~g}=\left(a_{d} / 1 \mathrm{~cm}\right) \times 3.0 M_{\oplus} .
$$


For the all particle size to be at the "characteristic" size $a_{c}=1-2 \mu m$, one would need $M_{\text {char }} \sim 3-6 \times 10^{-4} M_{\oplus}$.

If the IR emitting particles are smaller than both $a_{1}$ and $a_{2}$ they would be blown away from the ring region on a dynamical time-scale $\tau_{d}=\Omega^{-1} \sim 10^{2} \mathrm{yr}$ in both gas-rich and gas-free environment. Over the life span of HR4796A $\left(\tau_{\star}=8 \times 10^{6} \mathrm{yr}\right)$, an amount $M_{\text {lost }}^{\text {char }}=M_{\text {char }} \Omega \tau_{\star} \sim 3-6 M_{\oplus}$ of characteristic-size particles is lost through blow out by radiation pressure. In order to replenish this loss, Wyatt et al. (2000) proposed that the IR-emitting $\mu m$-size particles are collisional fragments of larger parent particles. Unless we are observing HR 4796A in a special epoch, the total amount of mass in the parent particles $\left(M_{t o t}\right)$ is likely to be comparable to or larger than Mlost char.

In the model presented by Telesco et al. (2000), only a single population of characteristic $\mu \mathrm{m}$-size particles is assumed to be responsible for the reprocessing of stellar radiation. In order for the parent particles assumed by Wyatt et al. (2000) to remain inconspicuous, their total surface area must be less than that of the $\mu m$-size IR-emitting particles. For a population of parent particles to contribute a major fraction of $M_{t o t}$, their total area is $\propto a_{d}^{-1}$. Since $M_{\text {lost }}^{\text {char }} / M_{\text {char }} \sim \Omega \tau_{\star} \sim 10^{5}$, the minimum size for the parent particles is $a_{\text {min }} \sim 10^{5} a_{c} \sim 10 \mathrm{~cm}$ such that their $\tau_{z}$ is less than that of the IR-emitting $\mu m$-size particles.

\subsection{Particle collisions}

The production of $\mu m$-size particles requires collisions. The collisional frequency for individual characteristic particles is

$$
\nu_{c} \sim n_{d} A_{d} \sigma_{d}
$$

The spatial number density of those particles which contribute to most of the IR radiation is

$$
n_{d}=\frac{M_{t o t}}{M_{d} 4 \pi R d R H_{d}} \simeq \frac{2}{3 \pi} \frac{L_{d i s k}}{L_{*}} \frac{\rho_{d} a_{d}}{m_{d} H_{d}} .
$$

For photons with wavelength smaller than the characteristic size of the particles, the particles' opacity is determined by their geometric cross section $A_{d}$. The optical depth in the direction normal to the plane of the disk is $\tau_{z} \sim\left(M_{\text {char }} / m_{d}\right)\left(A_{d} / \pi R^{2}\right) \sim L_{\text {disk }} / L_{\star}$. From Eqs. (23) and (25) we find the collisional frequency for IR emitting particles to be

$$
\nu_{c} \sim \nu_{c I}=\frac{R}{4 \pi d R} \frac{L_{d i s k}}{L_{\star}} \Omega<\tau_{z} \Omega<<\tau_{d}^{-1} .
$$

If the dominant contributors of the IR radiation are $\mu m$-size particles as postulated by Wyatt et al. (2000), they would be blown out on a dynamical time-scale because their $a_{d}<a_{1}$ (in a gas-free environment) and $a_{d}<a_{2}$ (when the particles are embedded in a gaseous disk). Thus, after they

are produced from the collisions of their parent particles, these particles would not have sufficient time to collide, fragment, or coagulate among themselves before they are ejected of the ring region. 
Furthermore, in order for the $\mu m$-size particles to remain as the dominant IR emitters, both $\tau_{z}$ and $\nu_{c}$ must decrease with particle size so that the particles with $1-2 \mu m<a_{d}<a_{1}$ or $a_{2}$ would not dominate the radiation reprocessing. Since both $a_{1}$ and $a_{2}$ are considerably larger than a few $\mu m$, the hypothetical $\mu m$-size IR-emitting particles must be produced directly (rather than through a cascade process) during collisions between the smallest surviving particles (those with $a_{d}>a_{1}$ or $\left.a_{d}>a_{2}\right)$.

We now consider the collision frequency of the population of parent particles which are the main suppliers of the $\mu m$-size IR-emitting particles. In the minimum-size (with $a_{d} \sim a_{\text {min }} \sim 10$ $\mathrm{cm})$ limit, the parent particles' $\nu_{c}$ is comparable to that of the IR-emitting particles, i.e. $\nu_{c I}$ in eq(27). On the dynamical time-scale during which the $\mu m$-size particles are blown away, a total fraction $\nu_{c I} \tau_{d}$ of the parent particle population would collide. In order for this limited number of events to directly replenish the mass low due to the radiative blow-away of the IR-emitting particles, each collision must generate $N_{f} \sim\left(\tau_{\star} / \tau_{d}\right)^{2} L_{\star} / L_{\text {disk }} \sim 10^{12}$ ( $\mu m$-size) fragments. The parent bodies could have a size $a_{d}>a_{\text {min }}$, in which case, collisions would occur less frequently by a factor $\sim a_{\text {min }} / a_{d}$ provided they can lead to the production of $\sim\left(\tau_{\star} / \tau_{d}\right)^{2}\left(a_{d} L_{\star} / a_{\text {min }} L_{\text {disk }}\right)$ fragments. Note that particles with sizes $>\left(\tau_{\star} L_{\text {disk }} / \tau_{d} L_{\star}\right) a_{\text {min }}$ collide less than once during $\tau_{\star}$. Nevertheless, at least in principle, collisions among a fraction of the large-particle population may replenish the $\mu m$-size IR-emitting particles.

The considerations presented in this subsection indicate that the $\mu m$-size particle scenario suggested by Telesco et al. (2000) and Wyatt et al. (2000) requires not only a population of parent bodies which are larger than a few $\mathrm{cm}$ but also a non continuous bimodal $(\sim 1 \mu \mathrm{m}$ and $>10 \mathrm{~cm})$ particle-size distribution. There is no simple physical mechanism to preferentially produce this type of particle distribution function.

\subsection{Particle size distribution}

In reality, collisions are more likely to produce fragments with a range of sizes. As noted above that all fragments with $a_{d}>a_{1}$ (in a gas-free environment) or $a_{d}>a_{2}$ (if the fragments are embedded in a residual gaseous disk) would survive the radiative blow out. During the life span of HR4796A, the minimum-size parent particles would undergo $\nu_{c I} \tau_{\star} \sim\left(L_{\text {disk }} / L_{\star}\right)\left(\tau_{\star} / \tau_{d}\right) \sim 10^{3}$

collisions. Although fragmentary particles with $a_{1}<a_{d}<a_{\min }$ or $a_{2}<a_{d}<a_{\text {min }}$ may collide less frequently, they may nevertheless attain a collisional coagulation-fragmentation equilibrium.

In principle, the particles' size distribution may be obtained by solving the appropriate coagulation equation with both coagulation and fragmentation effects included. Approximate solutions may be obtained for collisional steady state in which the disruption or growth rates $\left(\dot{M}_{d}\right)$ of the total mass $\left(M_{d}=N_{d} m_{d}\right)$ for a given population may be assumed to be independent of $a_{d}$. If we assume the size-distribution evolution occurs primarily through collisions between similar 
size particles,

$$
\dot{M}_{d} \simeq M_{d} \nu_{c} \sim N_{d}^{2} m_{d}\left(a_{d} / R\right)^{2} \Omega .
$$

A constant $\dot{M}_{d}$ would imply $N_{d} \propto a_{d}^{-2.5}, M_{d} \propto a_{d}^{0.5}$ and $\tau_{z} \propto a_{d}^{-0.5}$, i.e. most of the mass are contained in the large particles whereas most of the surface area cross sections are contained in the small particles. This particle size distribution is similar to that derived for collisional equilibrium (Hellyer 1970, Mathis et al. 1977) and adopted by Augereau et al. (1999) in which

$$
d N_{d} / d a_{d} \propto a_{d}^{-3.5}
$$

More generally, the growth and fragmentation may be mainly regulated by collisions between different-size particles, which would result in a slightly modified size distribution.

The distribution function in eq(29) is only applicable for particles with $a_{d} \sim a_{1}$ or $a_{d} \sim a_{2}$. Since smaller particles are radiative blow out of the ring before they can collide with each other again, their size distribution is probably determined by that resulting from the break up of the parent bodies. But if this distribution function can be extended to the small particles the total mass carried by the blown-away dust particles is $M_{\text {lost }}^{\text {tot }} \sim M_{\text {lost }}^{\text {char }}\left(a_{1} / a_{c}\right)^{1 / 2} \simeq 5-10 \times 10^{-4} M_{\oplus}$ in the gas free environment and $M_{\text {lost }}^{\text {tot }} \sim M_{\text {lost }}^{\text {char }}\left(a_{2} / a_{c}\right)^{1 / 2} \simeq 3-6 \times 10^{-3} M_{\oplus}$ if the particles are embedded in a protostellar disk of gas. Note that the inferred values of $M_{\text {lost }}^{\text {char }}$ and $M_{\text {lost }}^{\text {tot }}$ are comparable to Uranus and Neptune's masses and the Oort's cloud (Duncan et al. 1987). This mass is larger than the present mass estimate $\left(\sim 0.1 M_{\oplus}\right)$ for the Kuiper Belt today (Luu et al. 1997) but may be comparable to it in the past (Stern et al. 1997, Kenyon \& Luu 1999). The associated surface density of heavy elements is also comparable to that extrapolated from the minimum mass nebula for $R_{0}=70 \mathrm{AU}$.

\subsection{Preserved particle scenario}

We now consider the possibility that a range of particles were formed inside a gaseous protostellar disk. When the surface density of the disk is depleted, particles in the outer regions of the disk become exposed to the stellar radiation, all particles with $a_{d}<a_{2}$ would be blown away by the radiation pressure. If these small particles are not the dominant IR emitters, they would not not need to be continually replenished by some parent particle. In their model analyses of a large wavelength range of IR emission, Augereau et al. (1999) adopt a distribution of particles with sufficiently large size to survive radiation blow outs. We follow their approach by examining the evolution of the surviving particles.

Using our model parameters, we find $a_{2}=100-200 \mu m$ (see Fig. 1 and Fig. 3). (Note that $a_{2}$ does not directly depend on the gas surface density as long as $a_{0}>a_{2}$ ). For illustrative simplicity, we assume the IR-emitting particles have a characteristic size $a_{d}=a_{0}=600 \mu \mathrm{m}$ such that eq(24) gives $M_{\text {char }} \sim 0.18 M_{\oplus}$. Since these particles survive the radiation blow out, they collide up to $\sim \nu_{c} \tau_{\star} \sim\left(L_{d i s k} / L_{\star}\right)\left(\tau_{\star} / \tau_{d}\right) \sim 10^{3}$ times within the life span of the central star. 
In order to determine the collisional velocity among the particles, we note that in the absence of any warp, the optical depth in the disk's radial direction to the host star is $\tau_{r} \sim \tau_{z} R / H_{d}$. Since the surface brightness of the disk is spread out over an extended radial range, $\tau_{r}<1$ and $H_{d} / R>L_{d i s k} / L_{\star}$ for the IR emitting particles. The corresponding velocity dispersion of the particles would be $\sigma_{d} \geq 3 \times 10^{3} \mathrm{~cm} \mathrm{~s}^{-1}$. If these particles are embedded in a gaseous background,

$H_{d}$ is expected to be $\leq H_{p}$ which corresponds to an upper limit $\sigma_{d} \leq 10^{5} \mathrm{~cm} \mathrm{~s}^{-1}$. Within this velocity range, collisions may lead to either erosion (Hatzes et al. 1991) or coagulation if the particles are coated with frosts or traces of methanol as in comets Supulver (et al. 1997). Repeated collisions may eventually lead to an equilibrium in which fragmentation and coagulation are balanced to result in a size distribution similar to that in eq(29) (see review by Artymowicz 1997).

In addition to the mass requirement, a population of surviving particles (with $a_{d}>a_{1}$ and $a_{d}>a_{2}$ ) need to have a spatial distribution similar to that observed ring structure whether they are parent particles or the dominant IR emitting particles. In the following subsection we show that particles which are marginally larger than $a_{2}$ can congregate into rings if there are some non monotonic variations in the $\Sigma$ distribution of the gas. However, if particles become larger than $a_{0}$, they decouple from the gas flow. Nevertheless, this confinement mechanism continues to be effective provided $d V$ become positive although the rate of concentration decreases with the particle size. Meter sized particles need $\approx 10^{3}$ times longer to concentrate as they radially drift about $10^{3}$ times slower then the $600 \mu$ particles.

\subsection{A set of quasi-static models}

\subsubsection{Model: $A$}

We first consider the hydrostatic structure of both gas and dust disk. We set the initial surface density distribution of the gas to be proportional to $R^{-5 / 2}$ as it is typical for protostellar disks. The presence of gas is invoked in order to establish a flow with velocity reversal which would spontaneously lead to the emergence of ring structure. In $\S 2.1$, we adopt the surface density distribution of the gas disk to be that in eq (1) with its mass within $70 \pm 20$ AU to be $99 M_{\oplus}$ in order to augment for the solar composition. As a free parameter, we set $d=0.9$ which corresponds to a smooth $10 \%$ surface density increase at $70 \mathrm{AU}$. The amplitude of this variation in $\Sigma$ (see Fig. 1 and Fig. 3) is much smaller than that associated with gap formation.

The disk structural parameters are calculated self-consistently for a dynamically stable, rotating, optical-thin disk. This equilibrium configuration is established on the dynamical time-scale $\tau_{d}$. The increase in surface density of the gas at $70 \mathrm{AU}$ corresponds to a minor peak in density, a local minimum in the friction time and stokes number, but it has no effect on temperature and pressure scale height, because the disk is optical thin (Fig. 1). The pressure as function of density also shows a small amplitude local maximum. The resultant local maximum in the radial pressure gradient strongly influences the local rotational profile such that the deviation 
from the Keplerian rotation speed is decreased by $50 \%$. The velocity resulting from the Poynting Robertson effect is orders of magnitude smaller and it attains this local minimum as a consequence of the decrease in the friction time $\tau_{f}$ (see eq. 5). The last frame shows the minimum radius for particles to be gravitationally bound $\left(a_{2}\right)$, which is given in eq(18) by the equilibrium by the radiation pressure, gravity and centrifugal forces. This plot also indicates the upper particle size limit for particles to be stopped at $70 \mathrm{AU}$, which is about $700-800 \mu$.

For these disk parameters, our chosen particle's size is $\left(a_{d}=a_{0}=600 \mu \mathrm{m}\right)$ to receive maximal velocities and thus to show the upper limit of concentration. In Fig. 2, the solid line represents the resulting drift velocity of these particles due to combined effect of gas drag and radiation pressure in accordance to eq(15). In order to consider the relative importance of various contributions, we compare our main results with the dashed-line velocity curve for particles in a gravity and rotation free system, where radial radiation pressure and friction forces cancel each other. In this idealized case, the particles' radial velocity is positive everywhere because the friction time grows faster than the radiation pressure decreases with radius. The decline in $v_{r}$ at $70 \mathrm{AU}$ is due to the local minimum in the friction time-scale.

We also show with the dotted line, the $v_{r}$ distribution in a system where the radiation pressure is neglected and the orbital evolution is determined by gas drag only. In this limit, the radial drift is proportional to the friction time and the particles migrate inward everywhere. Due to the non monotonic variation in our prescribed $\Sigma$ distribution near $70 \mathrm{AU}$, the deviation of the gas velocity from the Keplerian flow is at a minimum. This change in $d V$ leads to a large increase in $v_{r}$. But with $d=0.9$ and $\delta R_{0}=20 A U$ in Model A, gas drag alone is not sufficient to change the sign of $d V$ and $v_{r}$. In contrast, when the effects of radiation pressure and gas drag are fully taken into account simultaneously, a radial velocity inversion is established near $70 \mathrm{AU}$.

For this model, particles with $a_{d}<a_{2}$ are blown away by the radiation pressure on the dynamical time-scale $\tau_{d}$. When the surface density of the gas is reduced below $\Sigma_{2} \equiv 3 L_{\star} /\left(8 \pi R c c_{s}^{3} \partial \ln \rho / \partial \ln R\right) \sim 0.1 \mathrm{~g} \mathrm{~cm}^{-2}, a_{0}$ becomes smaller than $a_{2}$ (see eq. 6 and eq. 18) and there are no longer particles that drift radially outward due to the combined forces from gas and radiation. If the motion of the disk gas is only affected by a negative pressure gradient, all particles with $a_{d}<a_{0}$ would be blown away by the stellar radiation pressure and all particles with $a_{d}>a_{0}$ would become segregated from the motion of the gas, drift inwards with a radial velocity in accordance to eq(17) and a corresponding orbital evolution time-scale $R / v_{r} \sim\left(R / H_{p}\right)^{2}\left(a_{d} \rho_{d} / \Sigma\right) \tau_{d} \sim\left(R / H_{p}\right)^{2} \tau_{d}$. In this case, all particles with sizes smaller than $\sim a_{2}\left(H_{p} / R\right)^{2}\left(\tau_{\star} / \tau_{d}\right)$ would be depleted during the lifespan of HR4796A. But $\Sigma_{2}$ is only marginally larger than the surface density of the parent particles inferred in $\S 3.1$. In $\S 2.4$, we have already shown that the particles' drag have a negligible effect on the motion of the gas. Eventually, when the surface density of the gas is reduce below $\Sigma_{1} \equiv 3 L_{\star} /\left(8 \pi G M_{\star} c\right) \sim 10^{-2} \mathrm{~g} \mathrm{~cm}^{-2}, a_{0}<a_{1}$. All particles with $a_{d}<a_{1}$ are blown away by the radiation pressure whereas larger particles would not undergo further orbital decay as the effect of head wind diminishes. 


\subsubsection{Model: $B$}

We also computed a model with the same $d$ but a smaller $\delta R_{0}=10 A U$ which represents a gaseous ring with a 2 times steeper surface density gradient (see Fig. 3). In this case, the gas rotation is hyper Keplerian with a sign change in $d V$ such that gas drag alone may lead to an outward velocity near $70 \mathrm{AU}$. All particles with $a_{d}>a_{2}$ or $a_{d}>a_{0}$ would migrate outwards in regions with a positive pressure gradient and inwards in regions with a negative pressure gradient. In this case there is no upper limit for the particle size in order to be stopped at 70 AU. Fig. 5 shows that the resulting drift velocities for $1 \mathrm{~m}$ sized objects is about three orders of magnitude smaller which nevertheless allows these bodies to concentrate in reasonable time in a ring at 70 AU.

\subsection{Dynamical evolution}

The resulting radial drift velocity (Eq. 15) can be used with the continuity equation to compute the global evolution of the dust surface density $\Sigma_{d}$ due to their orbital migration. In a cylindrical coordinate system,

$$
\frac{\partial \Sigma_{d}}{\partial t}=-\frac{1}{R} \frac{\partial}{\partial r}\left(R j_{\Sigma}\right)
$$

The local flux of dust particles is partially contributed by the systematic radial drift $v_{r}$ (see Eq. 15). Small particles with $a_{d}<a_{0}$, are well coupled to the disk gas. If the disk gas is turbulent, as in accretion disks in many astrophysical contexts (cf. Lin \& Papaloizou 1996), these particles may undergo diffusion following the trace of turbulent eddies. For computational convenience, we describe the turbulent stirring as passive diffusion in the conventional Navier-Stokes equation such that

$$
j_{\Sigma}=\Sigma_{d} v_{r}-\frac{D}{R} \frac{\partial}{\partial r}\left(R \Sigma_{d}\right) .
$$

A similar approach was used by Morfill and Völk (1984) to study the reprocessing of meteorites in the solar nebula. Following Cuzzi et al. (1993), we assume the turbulent diffusivity $D=\nu / S c$ with the Schmitt number which describes the coupling between the particle and the gas is set to be $S c=(1+S t)^{1 / 2}$. For the gas turbulent viscosity, we adopt the ad hoc $\alpha$ prescription which is commonly used in accretion disks (Shakura \& Sunyaev 1973) such that

$$
\nu=\alpha c_{s} H_{p} .
$$

The Stokes number for particles is modified from that for a laminar disk (see §2.2.1) by

$$
S t=\tau_{f} \omega_{t} .
$$

$\omega_{t}$ is the circular frequency of the largest eddy (typically smaller than $\Omega$ ). Thus, from the definition of the effective viscosity it follows:

$$
\omega_{t}=\frac{\nu}{H_{p}^{2}}
$$


For the particle size we are considering, the turbulent Schmitt number is $\sim 1$ so that maximum mixing is expected to occur.

The differential equation (30) is solved numerically using a finite volume scheme. It is integrated explicitly in time with a simple Euler backward scheme.

\subsubsection{Model: A}

In Figure 6 the initial radial $\Sigma_{d}$ distribution of $600 \mu m$ dust particles $\left(=10^{-2} \Sigma\right)$ is shown with the solid line. The evolution of the $\Sigma_{d}$ distribution is shown with a series of dotted lines which represents time sequences separated by $10^{3} \mathrm{yr}$ between each line. After the first $400 \mathrm{yr}$, the dust particles inside 66AU drift inward while that between 66 and $69 \mathrm{AU}$ drift outward. The lack of replenishment causes $\Sigma_{d}$ interior to $66 \mathrm{AU}$ to decline. The results in Figure 2 indicate that $v_{r}$ is a decreasing function of $R$ (i.e. the magnitude of $v_{r}$ is more negative at large $R$ ) inside 66 AU. Consequently, the depletion of the disk is more rapid at larger $\mathrm{R}$ and $\Sigma_{d}$ becomes a decreasing function of $R$ in this region. The outward drift at 66-69AU and inward drift at radius larger than 69AU cause the accumulation of dust particles between 67-73 AU and the depletion of dust exterior to $73 \mathrm{AU}$. After several $10^{3} \mathrm{yr}$, an equilibrium is reached in which the turbulent diffusion is balanced by the systematic drifts. In this equilibrium, $\Sigma_{d} R$ between $67-73$ AU becomes nearly two orders of magnitude larger than that of the disk interior to $67 \mathrm{AU}$ and another two orders of magnitude larger than that of the disk beyond $73 \mathrm{AU}$. The actual width of the ring depends on the magnitude of the effective viscosity parameter. A much wider ring may be obtained for a larger viscosity $\alpha>10^{-4}$.

After the equilibrium is established, the persistence of the gas is not required. In $\S 2.2 .2$, we show that as $\Sigma$ decreases during the depletion of the disk, $a_{0}$ decreases and $\tau_{f}$ increase while $d V$ is not significantly modified. While $a_{d}$ is still small compared with $a_{0}$, the factor $\left(L_{\star} A_{d} / 4 \pi R^{2} m c+2 \Omega d V\right)$ in eq(15) remains unchanged. Although the magnitude of $v_{r}$ may

increase due to the weakening hydrodynamic drag effect, the locations for velocity reversal remain unaltered. Thus, dust particle concentration in the ring is not affected by the depletion of $\Sigma$. Although dust particles would generally migrate inwards after $a_{0}$ decreases below their size $a_{d}$, the drag time-scale becomes so large that the radial locations of the particles are preserved. Thus, this scenario only needs the gas to be present for the initial few $10^{4}$ yrs in order to produce the ring. Afterwards it can be evaporated or be viscously diffused, while the dust distribution remains intact.

\subsubsection{Model: $B$}

Similar model parameter are applied to a narrower ring ( with $\delta R_{0}=10 A U$ ). In this model, we examine the concentration of $1 m$-size large objects. Fig. 目 shows the same behavior like 
the smaller particles, but this time the process is about 1000 times slower. Nevertheless, the concentration process takes an order of magnitude less time than the life time of HR4796A.

\subsubsection{Model: $C$ and $D$}

So far the upper limit for the observed gas around HR4796A is 1-7 $M_{\oplus}$ (Greaves et al. 1999), which is 1-2 orders of magnitude less than we used for Model A and Model B. Now we present to models with only $10 M_{\oplus}$ of gas (see fig. 8) and $1 M_{\oplus}$ (see fig. 10). In comparison to the first models the densities are smaller, the Stokes numbers for $600 \mu$ particles are bigger and thus the resulting drift velocities become also smaller. There are no significant changes in the critical particle size $a_{2}$.

Thus the dynamical evolution takes 5 times longer for Model C (see fig. 9) and about 25 times longer for Model D (see fig. 9). Even the time-scales increase at lower gas densities, the general effect persists.

\section{Summary and Discussions}

We examined in this paper the dynamics of the dust ring around HR 4796A with two separate scenarios. In the first scenario, we consider the case where the dominant emitters of the IR radiation are $\mu m$-size particles. Since these particles are being blown away by the radiation pressure of the central star, they must be replenished by large particles which are not affected by such a process. In a gas-free case, the parent particles must have a size greater than 10-20 $\mu \mathrm{m}$ whereas in a gas-rich environment, another order of magnitude larger size is needed. In order to account for the magnitude of reprocess radiation, $M_{\text {char }} \sim 3-6 \times 10^{-4} M_{\oplus}$ of $\mu \mathrm{m}$-size particles are needed at any given time. During the life span of HR4796A, a total mass $M_{\text {lost }}^{\text {char }} \sim M_{\text {char }} \tau_{\star} / \tau_{d} \sim 3-6 M_{\oplus}$ is needed in the reservoir of parent particles. In order for the parent particles to be inconspicuous sources of IR radiation relative to the $\mu m$-size particles, a large fraction of $M_{\text {lost }}$ must be contained in particles with $a_{d}>10 \mathrm{~cm}$. However, the smallest surviving particles (with sizes $\sim 200 \mu m$ ), could provide adequate supplies of $\mu m$ size particles if a large fraction of their mutual collisions results in their total fragmentation.

The main difficulty of identifying small particles as the dominant contributors of the IR radiation is the requirement of a non continuous bimodal size distribution. This problem would be removed if the particles which emit most of the IR radiation are sufficiently large $(>100 \mu m)$ to survive the radiative blow out. In this case, a total mass $<0.1 M_{\oplus}$ is needed throughout the life span of HR 4796A. We suggest the preservation of these modest-size particles (whether they are parent particles or the dominant IR emitters) is due to the combined effect of radiation pressure and gas drag. 
We presented a detailed model for the formation of dust rings in circumstellar disks. Our model can account for rings with sharply defined edges if it is embedded in a gas disk. A very small

amount of inhomogeneity is needed to induce the congregation of the particles. This inhomogeneity can be the result of a planet, but it does not necessarily has to be so. Photo-evaporation, an inhomogeneous initial distribution of mass after the infall, or inhomogeneities in the radial viscous transport of material can also account for such an anomaly in the surface density distribution. Thus we conclude that dust rings with sharp structures are not necessarily good indicator for embedded planets.

We thank D. Trilling and P. Bodenheimer for useful conversation. This work is supported in part through National Science Foundation grant AST-9618548, NASA grants NAG5-4277, 4494, 7515, and a special NASA astrophysics theory programme which supports a joint Center for Star Formation Studies at NASA-Ames Research Center, UC Berkeley, and UC Santa Cruz.

\section{REFERENCES}

Artymowicz, P. 1997, Annual Review of Earth and Planetary Sciences, 25, 175

Beckwith, S.V.W., \& Sargent, A.I. 1993, in Protostars and Planets III, ed. E.H. Levy \& J.I. Lunine (Tucson: Univ. of Arizona Press), 521

Beckwith, S.V.W., \& Sargent, A.I. 1993, ApJ, 402, 280

Beckwith, S.V.W., Sargent, A.I., Chini, R.S., \& Güsten, R. 1990, AJ, 99, 924

Boss, A.P. 1998, ApJ, 503, 923

Burkert, A., \& Bodenheimer, P. 1996, MNRAS, 280, 1190

Cameron, A.G.W. 1978, Moon and the Planets, 18, 5

Chiang, E.I., \& Goldreich, P. 1997, ApJ, 460, 368

Cuzzi, J.N., Dobrovolskis, A.R., \& Champney, J.M. 1993, Icarus, 106, 102

Cuzzi, J.N., Lissauer, J.J., Esposito, L.W., Holberg, J.B., Marouf, E.A., Tyler, G.L., \& Boischot, A. 1984, in Planetary Rings, ed. R. Greenberg \& A. Brahic (Tucson: Univ. of Arizona Press), 1984, 73

Duncan, M., Quinn, T., \& Tremaine, S. 1987, AJ, 94, 1330

Elliott, J.L., \& Nicholson, P.D. 1984, in Planetary Rings, ed. R. Greenberg \& A. Brahic (Tucson: Univ. of Arizona Press), 1984, 25

Goldreich, P., \& Tremaine, S. 1978, Icarus, 34, 240 
Goldreich, P., \& Tremaine, S. 1979, Nature, 277, 97

Goldreich, P., \& Tremaine, S. 1982, ARA\&A, 20, 249

Grün, E., Zook, H.A., Fechtig, H., \& Giese, R.H. 1985, Icarus, 62, 244

Hartmann, L., Calvet, N., Gullbring, E., \& D’Alessio, P. 1998, ApJ, 495, 385

Hatzes, A.P., Bridges, F., Lin, D.N.C., \& Sachtjen, S. 1991, Icarus, 89, 113 Goldreich, P., \& Tremaine, S. 1982, ARA\&A, 20, 249

Hayashi, C., Nakazawa, K., \& Nakagawa, Y. 1985, Protostars and Planets II, ed. D.C. Black \& M.S. Mathews (Tucson: Univ. of Arizona Press), 1100

Hillenbrand, L.A., Strom, S.E., Calvet, N., Merrill, K.M., Gatley, I., Makidon, R.B., Meyer, M.R., Skrutskie, M.F. 1998, AJ, 116, 1816

Jayawardhana, R., Fisher, R.S., Hartmann, L.W., Telesco, C.M., Pina, R.K., \& Fazio, G. 1998, ApJ, 503, L79

Jensen, E.L.N., \& Mathieu, R.D. 1997, AJ, 114, 301

Jura, M., Zuckerman, B., Becklin, E.E., \& Smith, R.C. 1993, ApJ, 418, L37

Jura, M., Ghez, A.M., White, R.J., McCarthy, D.W., Smith, R.C., \& Martin, P.G. 1995, ApJ, 445,451

Klahr, H.H., \& Henning, Th. 1997, Icarus, 128, 213

Kenigl, A. \& Ruden, S.P. 1993, Protostars and Planets III, ed. E.H. Levy \& J.I. Lunine (Tucson: Univ. of Arizona Press), 641

Kenyon, S.J., \& Luu, J.X. 1999, AJ, 118, 1101

Korycansky, D.G., \& Papaloizou, J.C.B. 1995, MNRAS, 274, 85

Lin, D.N.C. 1981, A\&A, 246, 972

Lin, D.N.C., \& Bodenheimer, P. 1981, ApJ, 246, 972

Lin, D.N.C., \& Papaloizou, J.C.B. 1979, MNRAS, 188, 191

Lin, D.N.C., \& Papaloizou, J.C.B. 1993, Protostars and Planets III, ed. E.H. Levy \& J.I. Lunine (Tucson: Univ. of Arizona Press), 749

Lin, D.N.C., \& Papaloizou, J.C.B. 1996, ARA\&A, 34, 703

Luu, J., Jewitt, D., Trujillo, C.A., Hergenrother, C.W., Chen, J., \& Offutt, W.B. 1997, Nature, 387,573 
Lynden-Bell, D., \& Pringle, J.E. 1974, MNRAS, 168, 603

Morfill, G.E., \& Völk, H.J. 1984, ApJ, 287, 371

Mundy, L.G., Looney, L.W., Erickson, W., Grossman, A., Welch, W.J., Forster, J.R., Wright, M.C.H., Plambeck, R.L., Lugten, J., Thornton, D.D. 1996, ApJ, 464, 169

Morfill, G.E., \& Völk, H.J. 1984, ApJ, 287, 371

Nakagawa, Y., Sekiya, M., \& Hayashi, C. 1986, Icarus, 67, 375

Nelson, A.F., Benz, W., Adams, F.C., Arnett, D. 1998, ApJ, 502, 342

Pollack, J.B., Hubickyj, O., Bodenheimer, P., Lissauer, J.J., Podolak, M., \& Greenzweig, Y. 1996, Icarus, 124,62

Preibisch, T., Sonnhalter C., \& Yorke, H.W. 1995, A\&A, 299, 144

Ruden, S., \& Lin, D.N.C. 1986, A\&A, 308, 883

Ruden, S.P., \& Pollack, J.B. 1991, ApJ, 375, 740

Safronov, V.S. 1969, NASA TTF-677

Schneider, G., Smith, A.S., Becklin, E.E., Koerner, D.W., Meier, R., Hines, D.C., Lowrance, P.J., Terrile, R.J., Thompson, R.I., \& Rieke, M. 1999, ApJ, 513, 127

Shu, F.H., Johnstone, D., \& Hollenback, D. 1993, Icarus, 106, 92

Shu, F.H., Najita, J., Ostriker, E., Wilkin, F., Ruden, S. \& Lizano, S. 1994, ApJ, 429, 781

Smith, B.A., \& Terrile, R.J. 1984, Science, 226, 1421

Stauffer, J.R., Hart,mann, L.W., \& Navascues, D.B. 1995, ApJ, 454, 910

Stern, S.A., McKennon, W.B., \& Lunine, J.I. 1997, in Pluto and Charon, 605

Supulver, K.D., Bridges, F.G., Tiscareno, S., Lievore, J., Lin, D.N.C. 1997, Icarus, 129, 539

Telesco, C.M., Fisher, R.S., Pina, R.K., Knacke, R.F., Dermott, S.F., Wyatt, M.C., Grogan, K., Holmes, E.K., Ghez, A.M., Prato, L., Hartmann, L.W., \& Jayawardhana, R. 2000, ApJ, 528 , in press

Toomre, A. 1964, ApJ, 139, 1217

Weinberger, A.J. Becklin, E.E., Schneider, G., Smith, B.A., Lowrance, P.J., Silverstone, M.D., Zuckerman, B., \& Terrile, R.J. 1999, ApJ, 525, 53

Wetherill, G.W. 1980, ARA\&A, 18, 77 
Whipple, F.L. 1972, in From Plasma to Planet, Proceedings of the Nobel Symposium 21, ed. A. Elvius (New York: Wiley)

Weidenschilling, S.J. 1977, MNRAS, 180, 57

Wyatt, M.C., Dermott, S.F., Telesco, C.M., Fisher, R.S., Grogan, K., Holems, E., \& Pina, R.K. 2000, ApJ, in press

Yorke, H., \& Bodenheimer, P. 1999, ApJ, 525, 330

Yorke, H., Bodenheimer, P., \& Laughlin, G. 1995, ApJ, 443, 199

Zuckerman, B., Forveille, T., \& Kastner, J.H. 1995, Nature, 373, 494 
Fig. 1.- Model A: The radial distribution of surface density, density, temperature, friction time for $600 \mu$-size particles, the resulting Stoke-Number, pressure scale height, the speed of sound, the pressure, the radial pressure gradient, the deviation from the Kepler rotational profile, the Poynting-Robertson effect and the size of the smallest gravitationally bound particles. The physical parameters for this plot are given by the mass $\left(M_{\star}=2.5 M_{\odot}\right)$ and luminosity $\left(L_{\star}=35 L_{\odot}\right)$ of the central object. Additional model parameters include the location of the maximum in surface density at $70 A U$, the width of the inner edge as $\delta R_{0}=20 A U$ and the gas mass around the edge between $R_{0}-\delta R_{0}$ and $R_{0}+\delta R_{0}$ which is taken to be 100 earth masses.

Fig. 2.- Radial velocities distribution of $600 \mu$-size particles for the model in Fig. 1. The solid line gives the effective radial drift. The dotted line accounts only for the gas-drag effect and the dashed line denotes only for the radiation pressure effect.

Fig. 3.- Model B: The radial distribution of surface density, density, temperature, friction time for $600 \mu$-size particles, the resulting Stoke-Number, pressure scale height, the speed of sound, the pressure, the radial pressure gradient, the deviation from the Kepler rotational profile, the Poynting-Robertson effect and the size of the smallest gravitationally bound particles. The physical parameters for this plot are given by the mass $\left(M_{\star}=2.5 M_{\odot}\right)$ and luminosity $\left(L_{\star}=35 L_{\odot}\right)$ of the central object. Additional model parameters include the location of the maximum in surface density at $70 A U$, the width of the inner edge which is $\delta R_{0}=10 A U$ and the gas mass around the edge between $R_{0}-\delta R_{0}$ and $R_{0}+\delta R_{0}$ which is 100 earth masses.

Fig. 4. - Radial velocity distribution of $600 \mu$-size particles for the model in Fig. 3. The solid line gives the effective radial drift.

Fig. 5.- Radial velocity distribution of $1 \mathrm{~m}$-size particles for the model in Fig. 3. The solid line gives the effective radial drift.

Fig. 6.- Model A:Evolution of the $600 \mu$-size particle surface density distribution for the model in Fig. 1. The solid line gives the initial distribution. The following lines are snapshots every 400 yrs.

Fig. 7.- Model A:Evolution of the $1 m$-size particle surface density distribution for the model in Fig. 3. The solid line gives the initial distribution. The following lines are snapshots every $10^{5}$ yrs.

Fig. 8.- Model C: The radial distribution of surface density, density, temperature, friction time for $600 \mu$-size particles, the resulting Stoke-Number, pressure scale height, the speed of sound, the pressure, the radial pressure gradient, the deviation from the Kepler rotational profile, the Poynting-Robertson effect and the size of the smallest gravitationally bound particles. The physical parameters for this plot are given by the mass $\left(M_{\star}=2.5 M_{\odot}\right)$ and luminosity $\left(L_{\star}=35 L_{\odot}\right)$ of the central object. Additional model parameters include the location of the maximum in surface density at $70 A U$, the width of the inner edge which is $\delta R_{0}=10 A U$ and the gas mass around the edge between $R_{0}-\delta R_{0}$ and $R_{0}+\delta R_{0}$ which is 10 earth masses. 
Fig. 9.- Model C: Evolution of the $600 \mu$-size particle surface density distribution for the model in Fig. 8. The solid line gives the initial distribution. Other lines are separated by a time interval of 2000 yrs.

Fig. 10.- Model D: The radial distribution for surface density, density, temperature, friction time for $600 \mu$-size particles, the resulting Stoke-Number, pressure scale height, the speed of sound, the pressure, the radial pressure gradient, the deviation from the Kepler rotational profile, the Poynting-Robertson effect and the size of the smallest gravitationally bound particles. The physical parameters for this plot are given by the mass $\left(M_{\star}=2.5 M_{\odot}\right)$ and luminosity $\left(L_{\star}=35 L_{\odot}\right)$ of the central object. Additional model parameters include the location of the maximum in surface density at $70 A U$, the width of the inner edge which is $\delta R_{0}=10 A U$ and the gas mass around the edge between $R_{0}-\delta R_{0}$ and $R_{0}+\delta R_{0}$ which is 1 earth mass.

Fig. 11.- Model D: Evolution of the $600 \mu$-particle surface density distribution for the model in Fig. 10. The solid line gives the initial distribution. The other lines are separated by time intervals of every $10^{4}$ yrs. 
Fig. 1
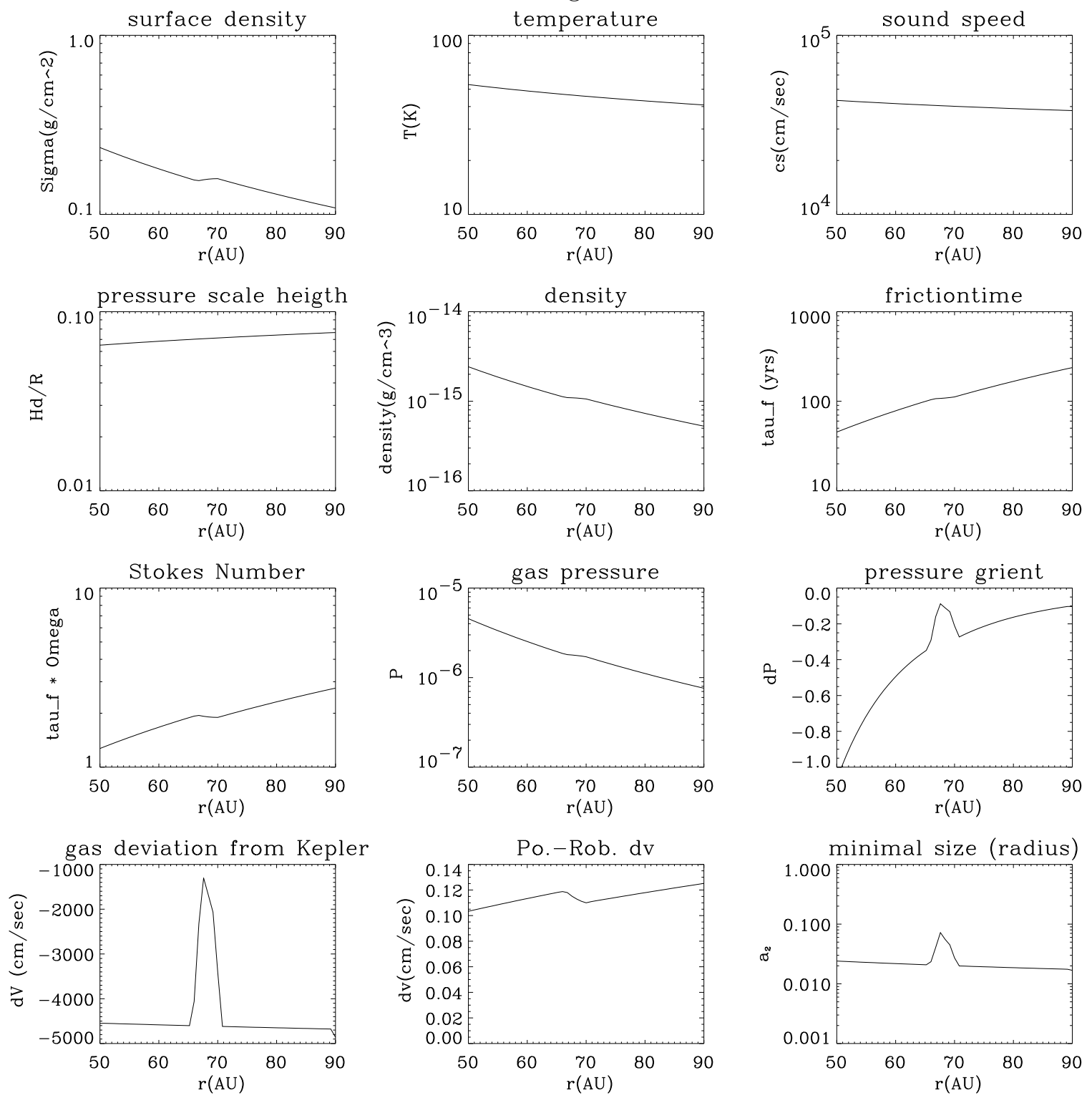
Fig. 2

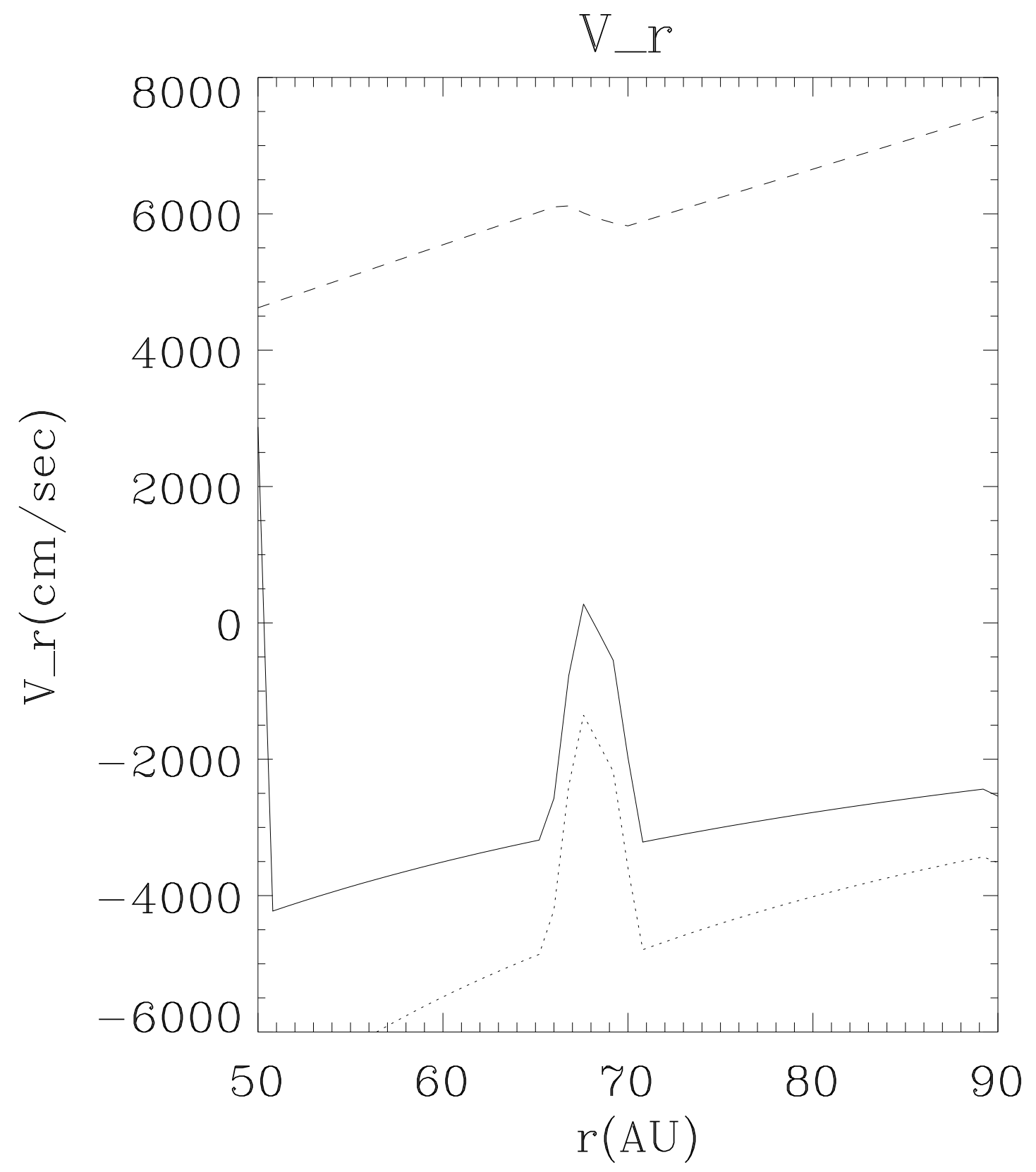


Fig. 3
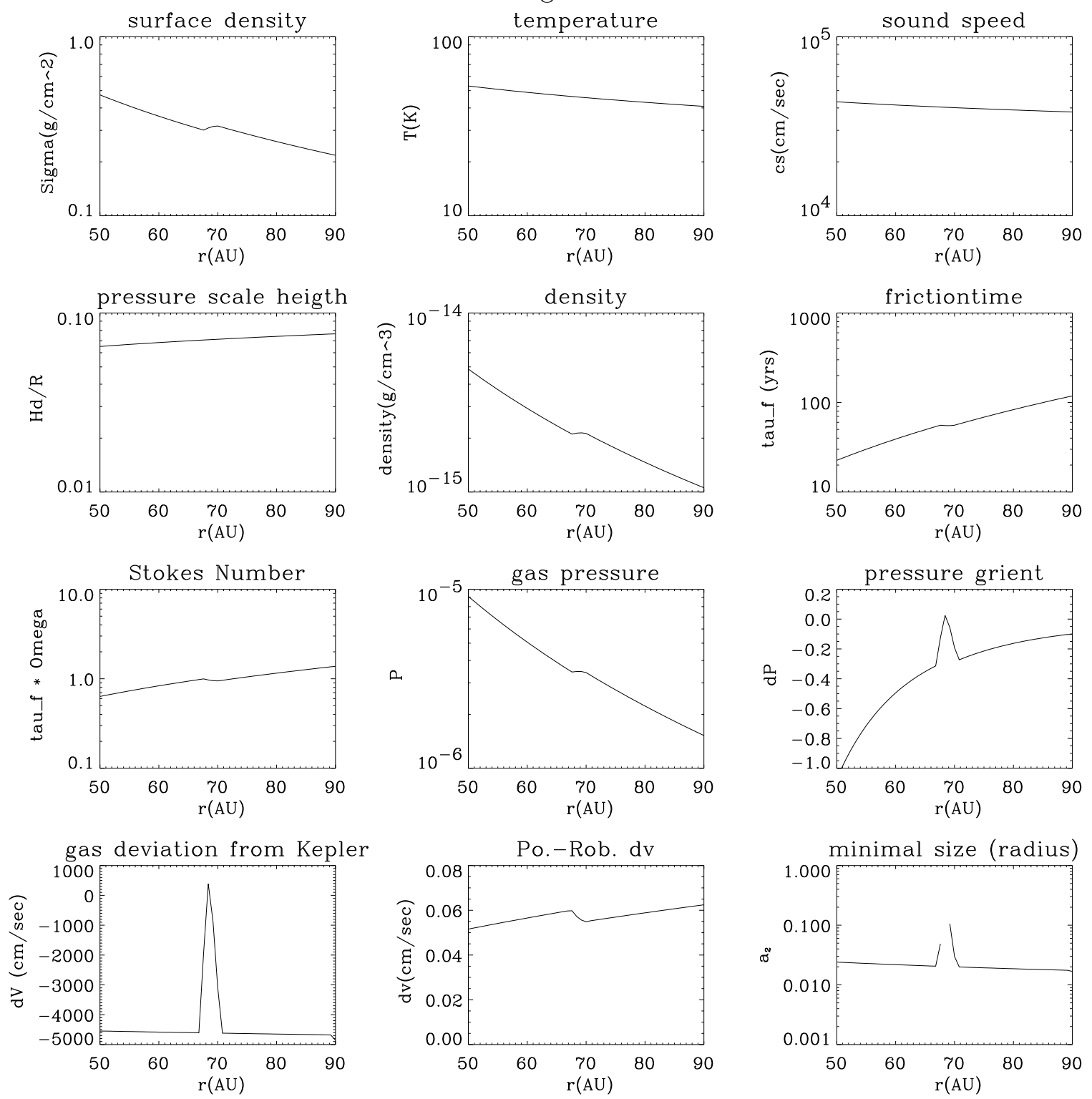
Fig. 4

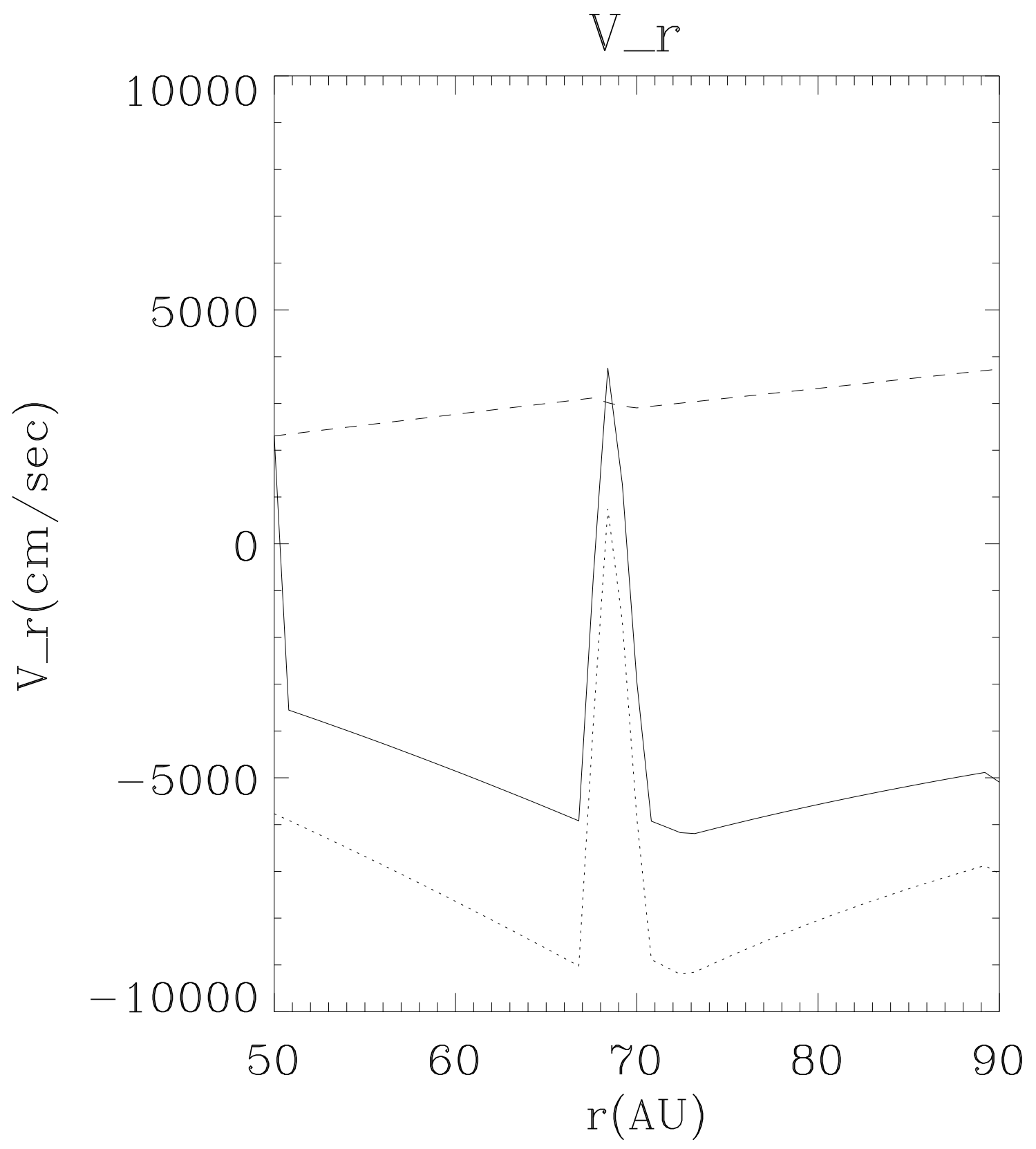


Fig. 5

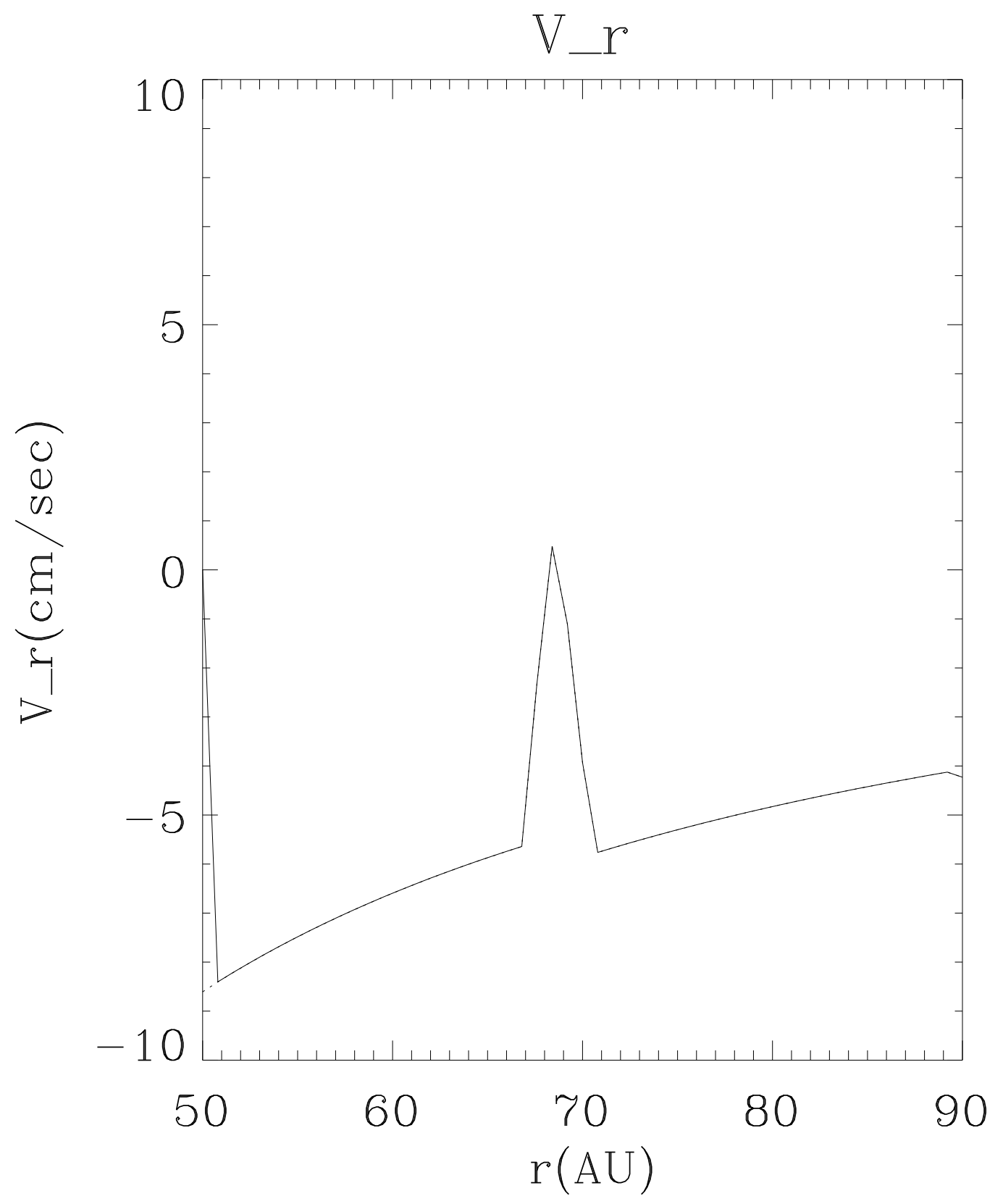


Fig. 6

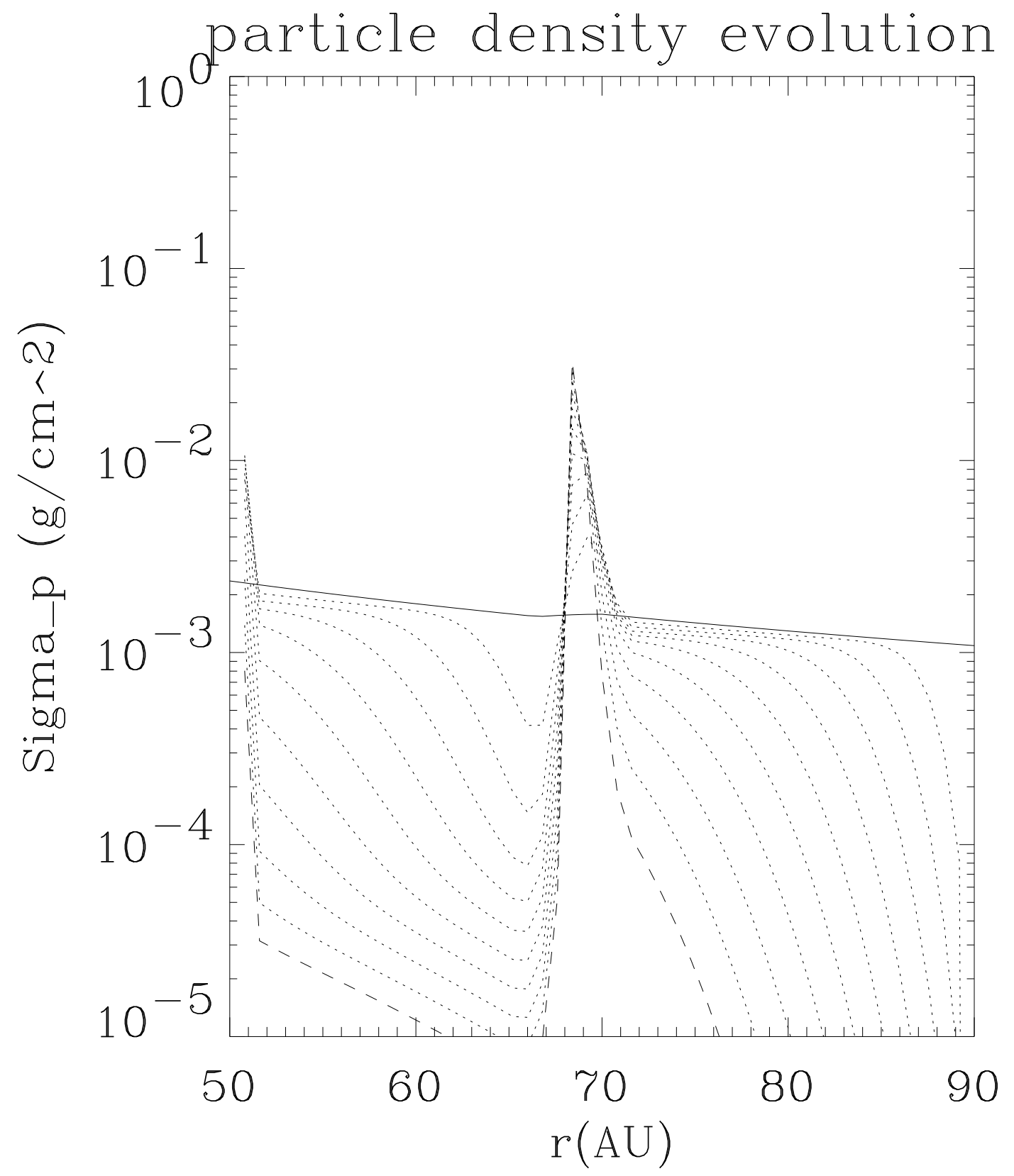


Fig. 7

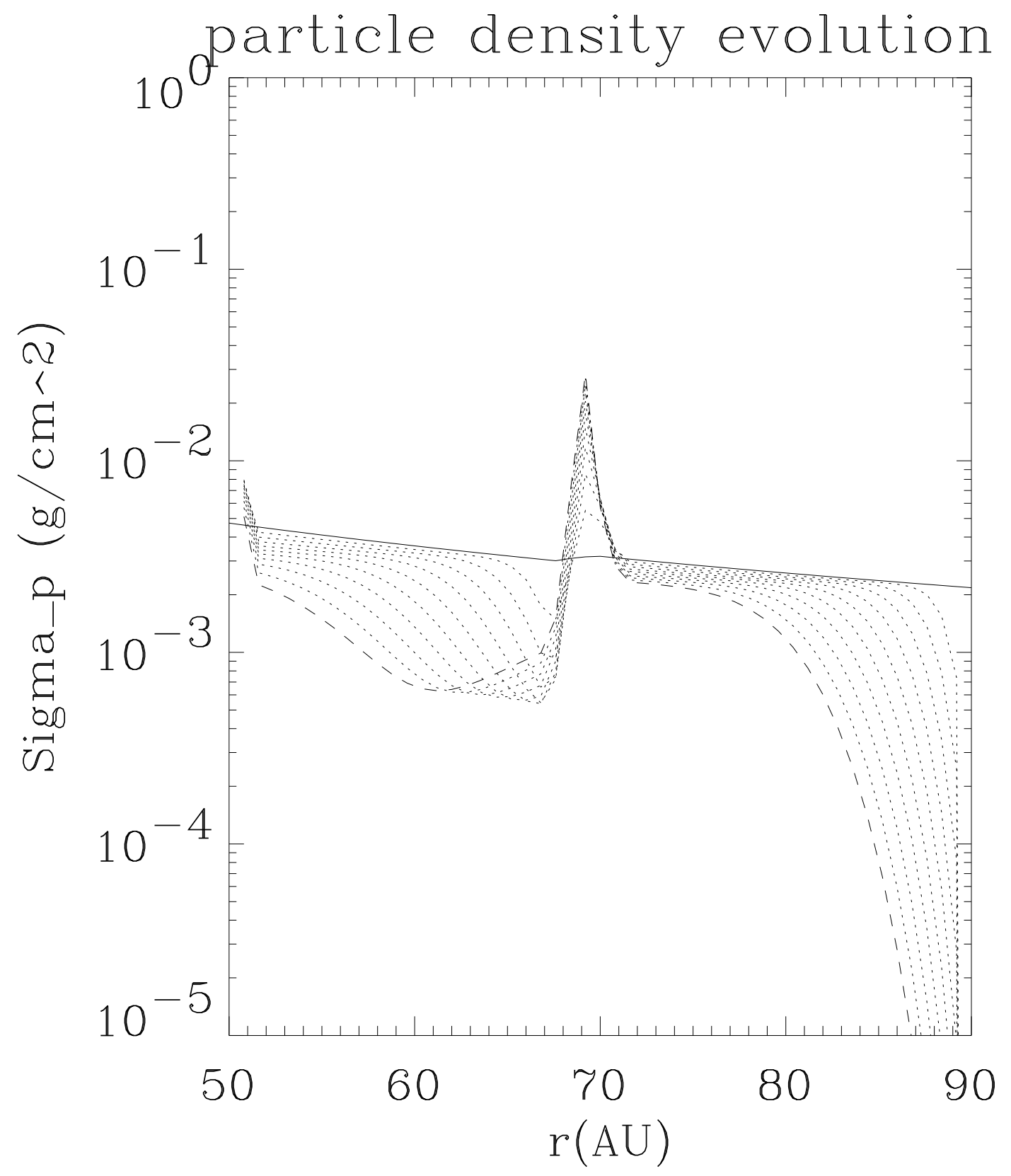


Fig. 8
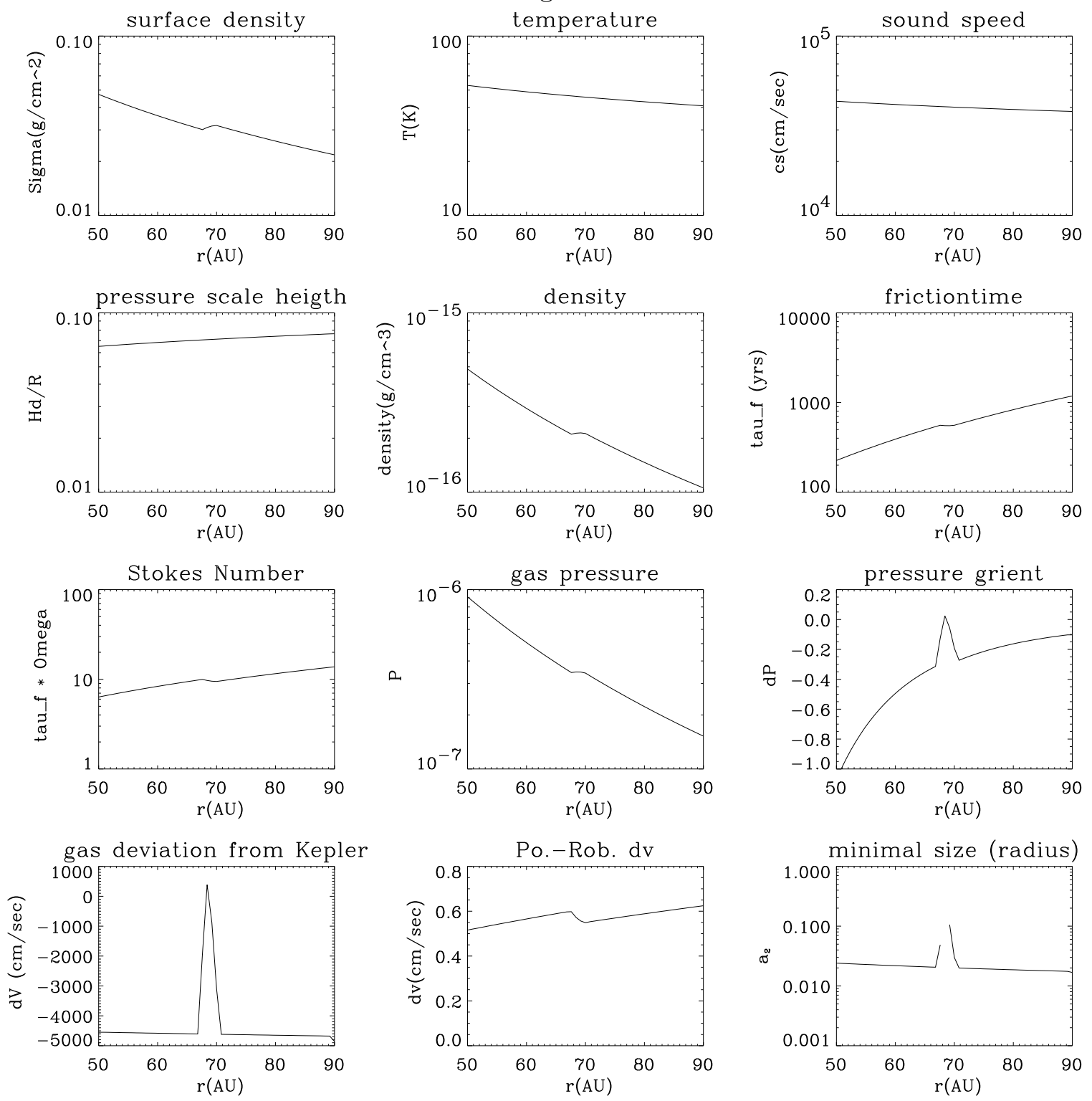
Fig. 9

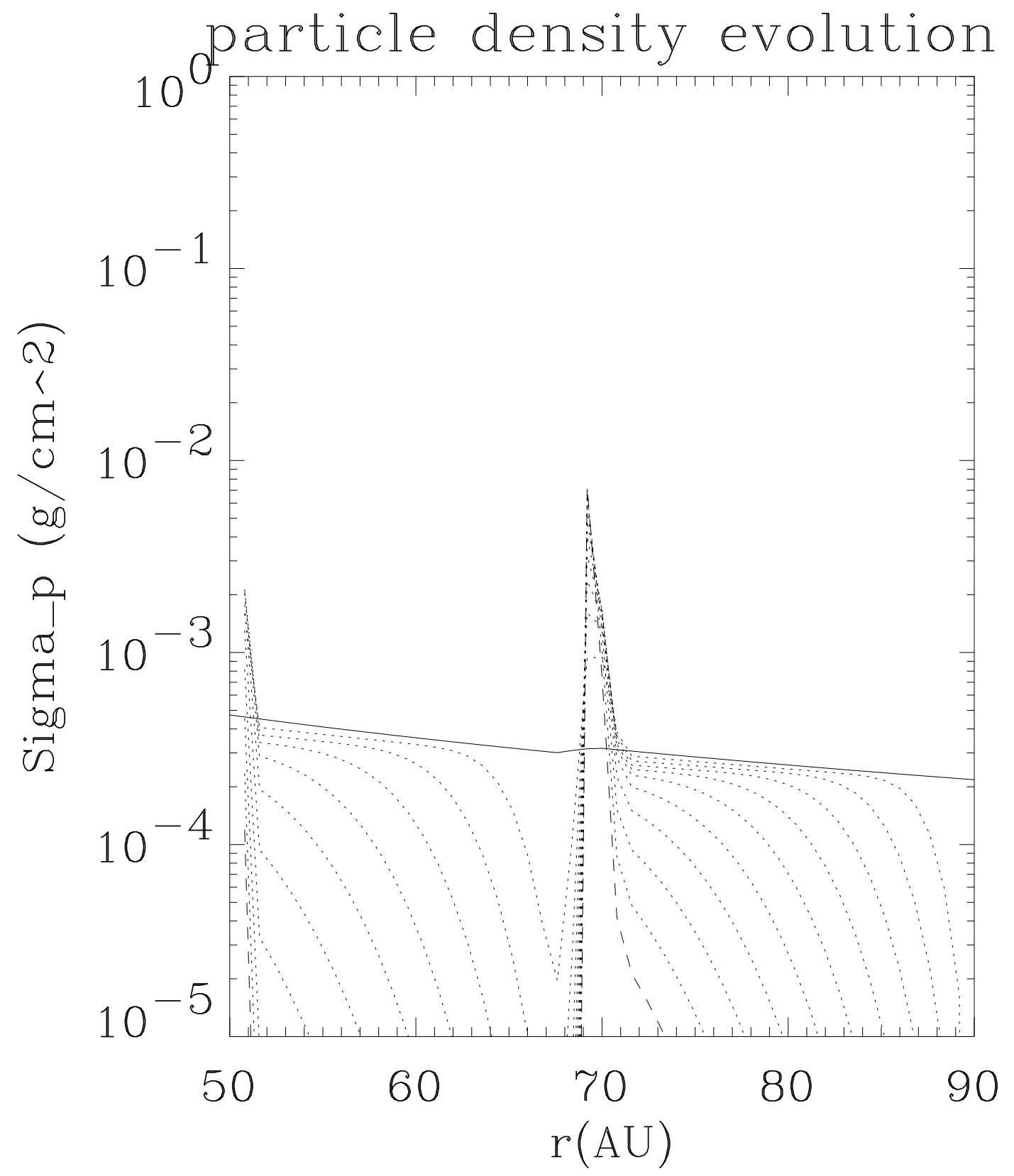


Fig. 10
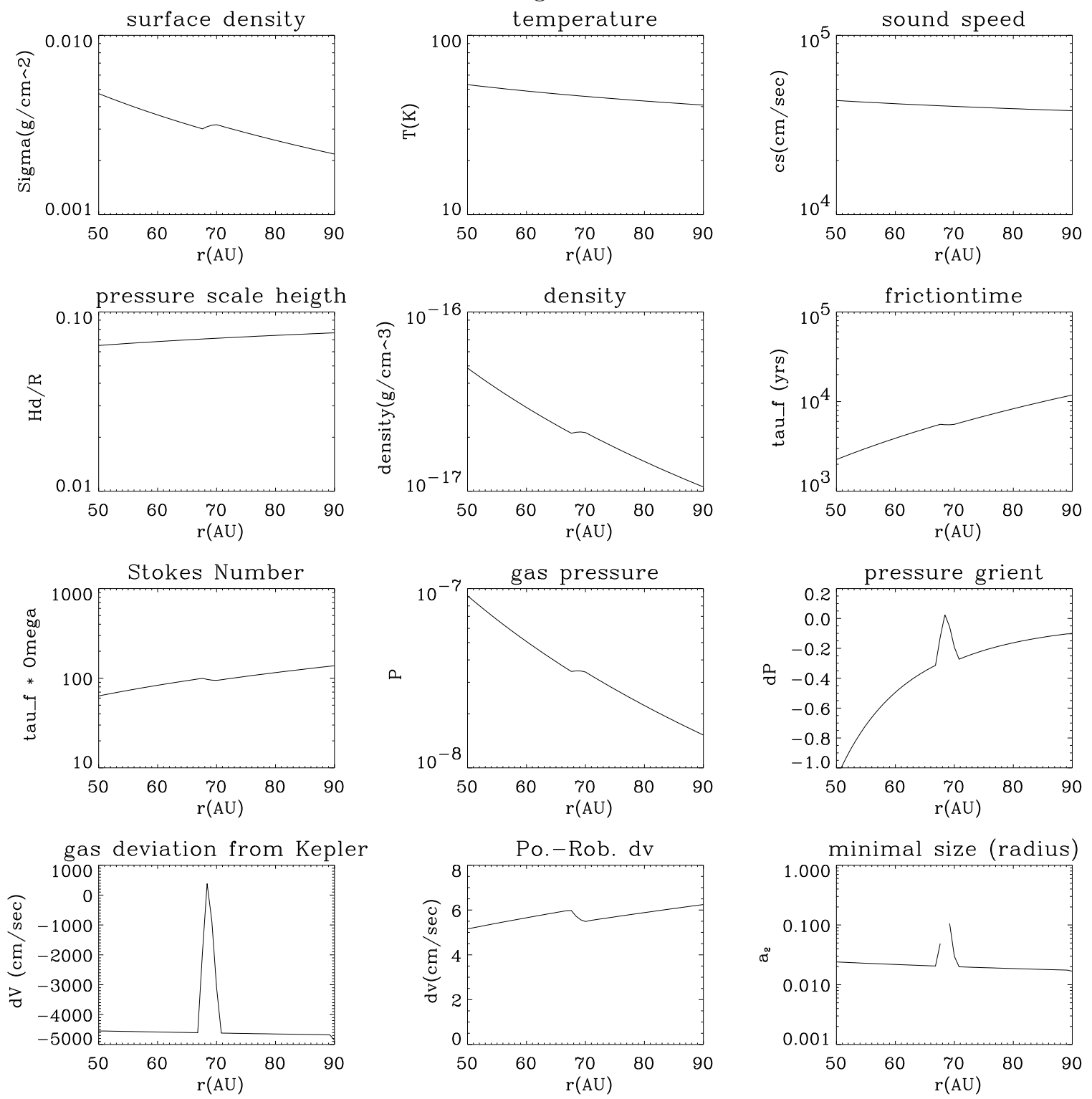
Fig. 11

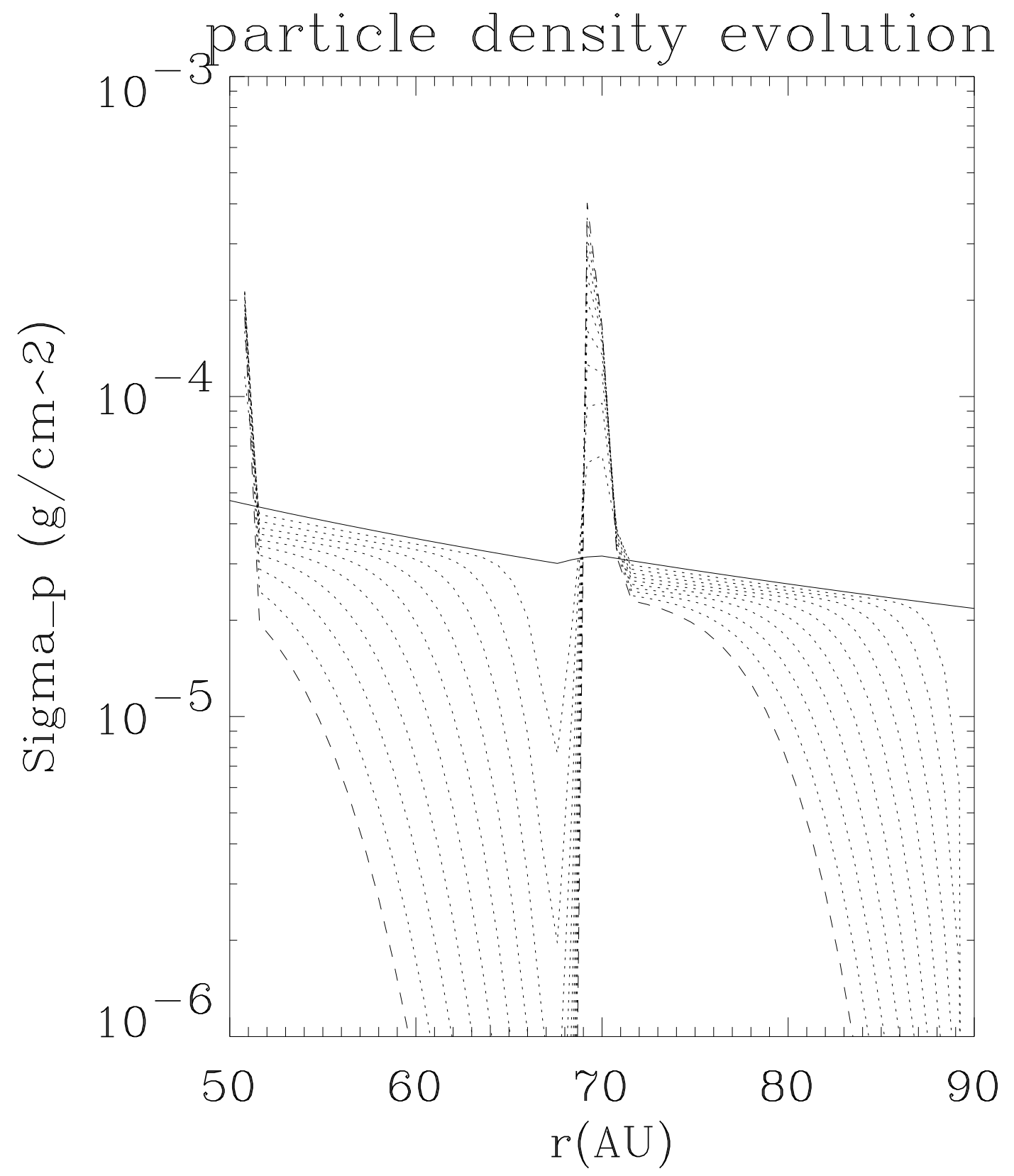

